\title{
1 Apical constriction induces tissue rupture in a proliferative epithelium
}

2

\author{
Mariana Osswald ${ }^{1,2}$, André Barros-Carvalho ${ }^{1,2}$, Ana M Carmo ${ }^{1,2,5}$, Nicolas Loyer ${ }^{3}$, Patricia C \\ Gracio $^{4}$, Claudio Sunkel ${ }^{1,2}$, Catarina C Homem ${ }^{4}$, Jens Januschke ${ }^{3}$ and Eurico Morais-de-Sá ${ }^{1,2, *}$ \\ 1. Instituto de Biologia Molecular e Celular, Universidade do Porto, Porto, Portugal. \\ 2. Instituto de Investigação e Inovação em Saúde (i3S), Universidade do Porto, Porto, \\ 3. Cell and Developmental Biology, School of Life Sciences, University of Dundee, Dow Street, \\ Dundee, DD5 1EH UK \\ 4. iNOVA4Health, CEDOC, NOVA Medical School, NMS, Universidade Nova de Lisboa, Lisbon, \\ Portugal. \\ 5. Present address: Institute of Biomedical Engineering, University of Toronto, Toronto, ON M5S \\ 3G9, Canada. \\ *Correspondence: eurico.sa@ibmc.up.pt
}

\begin{abstract}
Apical-basal polarity is an essential epithelial trait controlled by the evolutionarily conserved PAR-aPKC polarity network. Deregulation of polarity proteins disrupts tissue organization during development and in disease, but the underlying mechanisms are unclear due to the broad implications of polarity loss. Here, we uncovered how Drosophila aPKC maintains epithelial architecture by directly observing tissue disorganization after fast optogenetic inactivation in living adult flies and ovaries cultured ex vivo. We show that fast aPKC perturbation in the proliferative follicular epithelium produces large epithelial gaps that result from increased apical constriction, rather than loss of apical-basal polarity. Accordingly, we could modulate the incidence of epithelial gaps by increasing and decreasing actomyosindriven contractility. We traced the origin of epithelial gaps to tissue rupture next to dividing cells. Live imaging shows that aPKC perturbation rapidly induces apical constriction in nonmitotic cells, producing pulling forces that ultimately detach dividing and neighbouring cells. We further demonstrate that epithelial rupture requires a global increase of apical constriction, since it was prevented by the presence of non-constricting cells. Conversely, a global induction of apical tension through light-induced recruitment of RhoGEF2 to the apical side was sufficient to produce tissue rupture. Hence, our work reveals that the roles of aPKC in polarity and actomyosin regulation are separable and provides the first in vivo evidence that excessive tissue stress can break the epithelial barrier during proliferation.
\end{abstract}


49

50

51

52

53

54

55

56

57

58

59

60

61

62

63

64

65

66

67

68

69

70

71

72

73

74

\section{Introduction}

Cell polarity is a defining feature of epithelial architecture and function. Apical-basal polarity ensures the asymmetric localization of the intercellular junctions that maintain the cohesion of epithelia and thereby preserve the epithelial barrier. Epithelial architecture is also regulated by the distribution of actomyosin-driven forces at the apical, basal and junctional level $[1,2]$. It is thus not surprising that polarity disruption induces epithelial disorganization during animal development or disease [3-7]. This raises the importance of spatial cues provided by polarity regulators to build and support the three-dimensional structure of an organ. However, because polarity proteins are involved in many different processes that can ultimately affect tissue shape, how these proteins maintain epithelial architecture remains a critical longstanding question.

Interfering with polarity regulators in monolayered epithelia leads to different defects that disrupt epithelial integrity. These include the formation of multilayered tissue $[4,5,8]$, ectopic lumens $[5,9,10]$ and the appearance of gaps $[7,8,11,12]$. Extensive characterization using loss or gain of function perturbations linked these defects to junctional disorganization, misoriented cell division, defective control of proliferation or misdiferentiation. However, direct observation of how an epithelium becomes disorganized upon disruption of polarity regulators is still missing, which prevents a clear understanding of how defects arise.

Atypical Protein Kinase C (aPKC) is part of the apical PAR complex (Cdc42-Par6-aPKC) and is a central regulator of animal cell polarity[13,14]. It generates apical-basal asymmetry through phosphorylation of a number of polarity proteins, including Baz/Par3, Lgl, Par-1, Yurt and Crb. Their phosphorylation regulates local cortical binding through the modulation of multivalent protein interactions, homo-oligomerization [15-20], or simply by reducing electrostatic interactions with plasma membrane phospholipids [21, 22]. Apical-basal polarization ultimately positions belt-like adherens junctions (AJ) at the apical-lateral border where they mechanically link neighboring cells.

In addition to its well studied role in polarity regulation, aPKC regulates cell fate, epithelial-to-mesenchymal transition, cell cycle length, cell division orientation, actomyosin contractility and microtubule dynamics [9, 23-32]. In fact, some aPKC targets are not polarity proteins. Phosphorylation of Rho-associated coiled-coil-containing kinase (ROCK) represses the localization of this myosin activator to apical junctions, and thereby inhibits apical constriction in mammalian cells [26, 33, 34]. Moreover, aPKC acts as a negative regulator of apicomedial actomyosin networks to regulate pulsatile apical constriction in the Drosophila amnioserosa [35, 36]. aPKC function is also linked with actomyosin reorganization during cell division in fly tissues, which is consistent with its mitotic redistribution along the lateral cortex in mouse and sea anemone blastomeres [37-41]. Thus, aPKC may ensure epithelial architecture through different functional outputs, and assessing the contribution of actomyosin regulation and polarity maintenance demands separation of these aPKC functions.

Here, we combined optogenetic with chemical-genetic approaches to finetune aPKC inactivation with high-temporal control. This allowed us to disentangle the functions of aPKC in the regulation of actomyosin contractility and polarity. The monolayered follicular epithelium that encloses the Drosophila germline is a powerful system to explore the mechanisms that regulate epithelial architecture in vivo. Acute perturbation allowed us to capture that epithelial gaps form in the proliferative stages, where they arise by tissue rupture next to dividing follicle cells. We show this phenotype stems from the role of aPKC as an inhibitor of apical actomyosin networks in non-mitotic cells. These become hypercontractile after aPKC 
95

96

97

98

99

100

101

102

103

104

105

106

107

108

109

110

111

112

113

114

115

116

117

118

119

120

121

122

123

124

125

126

127

128

129

130

131

132

133

134

135

136

137

138

139

140

141 downregulation and pull on dividing cells until detachment occurs. Our work reveals the importance of keeping apical contractility in check during proliferation-mediated growth to maintain epithelial integrity.

\section{Results}

\section{Optogenetic clustering inactivates aPKC}

To explore how apical polarity maintains epithelial architecture, we developed a new approach to inactivate aPKC with high temporal control in the Drosophila follicular epithelium with an optogenetic clustering tool - light activated reversible inhibition by assembled trap (LARIAT) [42]. When exposed to blue light, LARIAT components - CRY2 fused to a GFP nanobody $\left(\mathrm{V}_{\mathrm{H}} \mathrm{H}\right)$ and $\mathrm{CIBN}$ fused to a multimerization domain - interact with each other and cluster. To target and sequester aPKC, we used flies with endogenously GFP-tagged aPKC [43] and co-expressed GAL4-driven UAS-LARIAT (UAS-V $\left.\mathrm{H}_{\mathrm{H}} \mathrm{H}: \mathrm{CRY} 2-\mathrm{P} 2 \mathrm{~A}-\mathrm{CIBN}: \mathrm{MP}\right)$ [44] specifically in the follicular epithelium (Figure 1A).

Expression of the UAS-LARIAT system in homozygous GFP::aPKC flies that remained in the dark did not interfere with aPKC localization or protein levels, and did not produce defects in epithelial organization (Figures $1 B$ and S1A). This shows that GFP::aPKC is fully functional in the presence of the LARIAT components. We then exposed female flies to blue light continuously for at least $24 \mathrm{~h}$ to test if optogenetic clustering reproduced the aPKC loss of function phenotypes described for the follicular epithelium [45, 46]. GFP::aPKC clustered in puncta, and led to the anticipated defects in epithelial architecture, namely epithelial gaps and multilayering (Figures $1 \mathrm{~B}$ and $1 \mathrm{C}$ ). A similar frequency of tissue defects was also visible after clustering heterozygous GFP::aPKC in the presence of an apkc mutant allele, but not in presence of the untagged wild-type allele (Figures 1B and 1C), which suggests that clustering inactivates GFP::aPKC. Furthermore, as predicted for aPKC inactivation, its substrate Lgl mislocalized to the apical domain upon aPKC optogenetic clustering in follicle cells (Figures $1 \mathrm{D}$ and $1 \mathrm{E}$ ). Taken together, these results show that illuminating flies is sufficient to trigger CRY2-CIBN heterodimerization and perturb aPKC with optogenetics in vivo in adult flies prior to dissection. We further evaluated the impact of optogenetic aPKC clustering in the assymetric distribution of Miranda during Drosophila neural stem cell division, where it is a relevant aPKC substrate [47]. aPKC clustering prevented Miranda's release from the apical domain of dividing larval neuroblasts (Figures S1B and S1C). Thus, LARIAT-mediated clustering is applicable to study aPKC in distinct contexts of cell polarity.

\section{Optogenetic aPKC inactivation leads to fast tissue disorganization}

We took advantage of optogenetic perturbation in vivo to monitor the progression of tissue disorganization in flies exposed to light for specific periods of time (Figures 2A and 2B). We narrowed the analysis to stages 4 to 6 of egg chamber development, so as to determine the impact of aPKC perturbation in epithelial architecture prior to major morphogenetic changes during egg chamber development. Multilayering was the most prevalent phenotype in fixed tissue from flies exposed to light for longer periods of time, whereas gaps were the most frequent defect upon 2 hours of GFP::aPKC clustering in vivo (2h: $\sim 50 \%$ egg chambers with gaps and $\sim 30 \%$ with multilayering; $4 \mathrm{~h}$ : $\sim 25 \%$ gaps and $\sim 85 \%$ multilayering; Figures $2 \mathrm{~A}$ 
and $2 \mathrm{~B}$ ). The two phenotypes were not mutually exclusive (Figure $1 \mathrm{C}$ ), and were commonly observed in different positions of the egg chamber (Figure 2C). Epithelial gaps appeared almost exclusively at the dorsal/ventral region, whereas multilayering was largely restricted to the egg chamber poles, which suggests a distict basis for the two phenotypes.

Intriguingly, gap frequency declined with increasing duration of light exposure (Figures $2 \mathrm{~A}$ and $2 \mathrm{~B}$ ), suggesting that epithelial gaps appear specifically during the initial phase of aPKC clustering and before the formation of multilayered tissue. We confirmed these results by live imaging using fluorescent markers of the nucleus (H2A::RFP) and plasma membrane in egg chambers cultured ex vivo. GFP::aPKC formed large clusters at the apical domain within minutes of exposure to blue light (488 nm; Figure 2D; Movie S1). Epithelial gaps formed within 30 minutes of light exposure and earlier than tissue multilayering (Figure 2D; Movie S1).

aPKC is likely only partially inactive during the initial period of clustering due to the time necessary to completely cluster and mislocalize aPKC. Prolonged aPKC clustering could futher inactivate $\mathrm{aPKC}$ and favor multilayering. To test if the predominance of distinct defects was associated with the level of aPKC inactivation, we treated ovaries mutant for an $a P K C$ ATP-analogue sensitive allele $\left(a P K C^{a s 4}\right)$ [48] for $2 \mathrm{~h}$ with a range of $1 \mathrm{NA}-\mathrm{PP} 1$ inhibitor concentrations. Treatment with $1 \mu \mathrm{M}$ of $1 \mathrm{NA}-\mathrm{PP} 1$, which in vitro reduces aPKC activity to $\sim 15 \%$ [48], led predominantly to epithelial gaps ( $50 \%$ of egg chambers with gaps vs $10 \%$ with multilayering), whereas increasing drug concentrations led predominantly to multilayering (Figures 2E and 2F). Thus, epithelial gaps are associated with partial aPKC inhibition, whereas multilayering arises after strong loss of aPKC function. Moreover, epithelial gaps are the earliest defect in epithelial architecture after aPKC inactivation.

\section{aPKC antagonizes apical constriction in multiple Drosophila tissues}

To identify the primary cellular effect underlying epithelial gaps, we clustered aPKC and analyzed the immediate impact on polarity, adhesion and the actomyosin cytoskeleton. In contrast to long-term clustering (Figure 1D), apical-basal polarity was not affected before gap formation, as both Lgl::mCherry and E-cad::mKate2 remained enriched at the lateral membrane and apical junctions, respectively (Figures $3 \mathrm{~A}$ and S2A). However, the fluorescence of Sqh::mKate2, a tagged version of non-muscle myosin II regulatory light chain (Myoll-RLC), increased rapidly at the apical side of the epithelium within minutes of lightexposure and prior to the formation of gaps (Figures 3B, 3C; Movie S2). Furthermore, this apical myosin increase was accompanied by an increase in circularity of the apical surface of the epithelium (Figures 3B and 3D). This tissue deformation could result from alterations in the apical area of individual cells. Accordingly, live imaging of mosaic epithelia with clonal expression of UAS-LARIAT showed that optogenetic aPKC clustering induced constriction of the apical area of LARIAT-expressing cells (Figures 3E and 3F; Movie S3). Thus, upregulation of apical contractility is the earliest effect upon optogenetic aPKC inactivation.

We further tested if the apical myosin increase was the first consequence of aPKC inactivation using chemical-genetics (Figures S2B and S2C). Live imaging showed that treatment of aPKC ${ }^{a s} 4$ egg chambers with $1 \mu \mathrm{M}$ 1NA-PP1 led to a quick increase of apical Sqh::mKate2, which persisted for $50 \mathrm{~min}$ in regions of the follicular epithelium without gaps. A higher inhibitor concentration (10 $\mu \mathrm{M}$ 1NA-PP1) also increased apical Sqh::mKate2 initially. However, this effect was transient, possibly due to the quicker loss of apical-basal polarity, which was reported to occur around 20 minutes after addition of $10 \mu \mathrm{M} 1 \mathrm{NA}-\mathrm{PP} 1$ in the 
follicular epithelium [48]. Perturbation of aPKC activity with high-temporal control therefore demonstrates that aPKC regulates apical contractility prior to polarity loss.

To determine if downregulation of apical myosin is a general function of aPKC, we analyzed the effect of aPKC inhibition on the neuroepithelium of the developing fly brain. Previous genetic perturbation in neuropithelial cells indicated that aPKC could support apical contractility by maintaining the polarized apical localization of myosin [28]. Therefore, we hypothesised that fast inactivation would also be necessary to separate the roles of aPKC in contractility and polarity in this tissue. We performed live imaging of $a P K C^{\text {as4 }}:$ :mScarlet brains to follow the initial impact of aPKC inhibition on apical shape and myosin accumulation. These experiments showed a dramatic constriction of the neuroepithelium within 10 minutes of inhibitor addition (Figures $3 \mathrm{G}$ and $3 \mathrm{H}$; Movie S4). Apical constriction was associated with an increase in junctional and medioapical myosin II intensity (Figures $3 \mathrm{I}$ and $3 \mathrm{~J}$ ). Hence, aPKC downregulates apical constriction to control the shape of distinct epithelial tissues.

\section{Epithelial gaps result from increased apical contractility}

To determine if increased apical actomyosin contractility is necessary to generate epithelial gaps in the follicular epithelium, we first disrupted the actin cytoskeleton with Latrunculin A (Lat A). Time-lapse imaging with E-cad::mKate2 to measure the apical area at the AJ level shows that treatment with LatA before light exposure blocks constriction during optogenetic clustering of aPKC (Figures $4 \mathrm{~A}$ and $4 \mathrm{~B}$ ). Moreover, disruption of the actin cytoskeleton prior to aPKC clustering prevented the formation of epithelial gaps in tissue exposed ex vivo for 2 hours to light (Figures 4C and 4D). To further test whether increased Myoll activity promotes gap formation, we modulated actomyosin contractility and evaluated the presence of epithelial gaps after in vivo aPKC clustering for 2 hours. Overexpressed unphosphorylatable (SqhAA) or phosphomimetic (SqhEE) versions of myosin-RLC respectively reduce and increase contractility when they form bipolar filaments with wild-type myosin-RLC [49-51]. Upon optogenetic aPKC inactivation, SqhAA overexpression restored epithelial integrity, whereas SqhEE overexpression increased epithelial gap frequency (Figure $4 \mathrm{E})$. Together, these results indicate that actomyosin-dependent cell contractility promotes and is necessary for the formation of epithelial gaps after aPKC inactivation.

\section{Epithelial gaps form by tissue rupture next to dividing cells}

During live imaging of Sqh::mKate2 in midsagittal egg chamber sections, we noticed that epithelial gaps frequently formed next to dividing cells (Figure S3A; Movie S5). To test if epithelial gaps appear specifically in proliferative tissue, we analysed egg chambers in stage 8 of oogenesis to ensure that they were not proliferating during the 2-hour period of lightinduced clustering. Epithelial gaps were almost absent in these non-proliferative stages (Figure S3B), which suggests that cell division challenges cell attachment in the follicular epithelium. Consistent with this, we observed frequent local loss of apical contacts next to dividing cells in aPKC ${ }^{\text {as }}$ neuroepithelia upon aPKC inhibition (Figure S3C), despite the absence of large tissue rupture. To address how cell division contributed to loss of tissue integrity, we imaged epithelial gaps forming in the follicular epithelium stained with a membrane marker. Strikingly, the majority of epithelial gaps initiated as ruptures between cells undergoing cytokinesis and their neighbors (Figures 5A and 5B; Movie S6) 
The force produced by cytokinetic ring constriction could promote epithelial rupture in the context of aPKC downregulation by pulling on neighboring cells undergoing apical constriction. To test whether cytokinesis was necessary for epithelial cells to detach from each other, we blocked cytokinesis either by disrupting contractile ring assembly with the Aurora $B$ inhibitor Binucleine 2 (Bin2) [52], or by blocking cells in prometaphase with the microtubuledepolymerizing drug Colchicine (Colch) (Figure S3D). We also depleted Tumbleweed/RacGAP50C (Tum; Figure S3E), a component of the centralspindlin complex that controls contractile ring assembly [53]. However, neither of these treatments prevented the formation of epithelial gaps upon aPKC clustering (Figures $5 \mathrm{C}-\mathrm{E}$ ). In addition, live imaging of egg chambers treated with Colch or Bin2 showed that optogenetic clustering of aPKC still led to recurrent tissue rupture next to mitotic cells even though they did not undergo cytokinesis (Figures $5 \mathrm{G}$ and $5 \mathrm{H}$ ). Altogether, we conclude that while tissue rupture upon aPKC perturbation is commonly observed next to dividing cells, it does not require cytokinesis. Thus, other aspects of cell division likely provide an additional challenge to epithelial upon increased apical constriction.

251

\section{Dividing cells are stretched by hypercontractile neighbors after aPKC inactivation}

To understand why epithelial gaps form between dividing and neighboring cells, we further characterized the impact of aPKC on Myoll distribution in mitotic and non-mitotic cells. During interphase, Myoll is enriched at the junctions and at the medioapical surface of follicle cells, where it drives pulses of apical constriction [54]. Live imaging showed GFP::aPKC accumulated at the apical intercellular contacts and displayed a smaller dynamic medioapical pool that accompanied the cycles of medioapical Myoll accumulation in interphasic follicle cells (Figures 6A and 6B; Movie S7). During mitosis, aPKC extends along the lateral cortex, a spatial redistribution that is also remininescent of Myoll (Figure 6A) [38]. To evaluate the effect of aPKC inactivation on distinct Myoll pools, we imaged Sqh::mKate2 in apical sections of epithelial cells. Myoll rapidly accumulated at the medioapical level upon optogenetic or chemical aPKC inhibition in interphasic follicle cells (orange arrows; Figures 6C and 6D; Movie S8). In contrast, in mitotic cells, aPKC inactivation did not affect the normal reduction of medioapical myosin (yellow arrows) nor the mitotic redistribution of myosin along the lateral cortex (blue arrow; Figures $6 \mathrm{C}$ and $6 \mathrm{D}$ ). This result suggests that aPKC is required to antagonize apicomedial actomyosin specifically during interphase.

We hypothesised that increased apical constriction in neighboring non-mitotic cells could produce excessive pulling on dividing cells, which could be unable to sustain this force due to the decrease in apical myosin. To evaluate this hypothesis, we monitored the effect of optogenetic aPKC inactivation on the apical surface area of mitotic cells with E-cad::mKate2. In contrast to interphasic cells (Figure 4A), mitotic cells did not contract, but rather expanded their apical domain upon mitotic entry and later detached from constricting neighboring cells (Movie S9). Quantification of the apical area in cells that were mitotic in the initial period of light exposure showed that clustering increased the expansion of the apical domain during mitosis (Figures 6E and 6F). Hence, excessive apical contractility in non-mitotic cells induces stretching of dividing cells and promotes tissue disruption.

\section{Increase in apical contractility at the tissue-level induces epithelial gaps}

To address whether rupture of the follicular epithelium was produced by a global or local increase in apical contractility, we analysed proliferative epithelial tissue with clonal expression 
of UAS-LARIAT. In contrast to egg chambers where the whole tissue expressed UAS-LARIAT, there was no rupture in mosaic egg chambers containing cells insensitive to light (Figures 7A and 7B), neither if LARIAT cells divided adjacent to wild-type cells $(n=16)$ nor within LARIAT clones $(n=28)$. This result suggests that the apical side of wild-type cells may stretch to accommodate the constriction of neighboring tissue and prevent tissue rupture. Accordingly, wild-type cells neighboring UAS-LARIAT patches expanded their apical area during light exposure (Figure 7C).

To test if the global increase of apical contractility is sufficient to drive tissue rupture, we used an optogenetic tool to stimulate apical constriction by light-dependent recruitment of the RhoGTPase activator RhoGEF2 (RhoGEF2::CRY2::mCherry) to an apically enriched PatJ::CIBN::GFP::CAAX fusion [55]. Live imaging showed cytoplasmic RhoGEF2::CRY2::mCherry was quickly recruited to the apical domain after light exposure (Figure 7D). Apical recruitment of RhoGEF2::CRY2::mCherry induced apical Myoll accumulation and produced gaps next to dividing cells (Figures 7D and 7E). Moreover, in vivo exposure of flies expressing this optogenetic system to $2 \mathrm{~h}$ of blue light reproduced the phenotype of aPKC LARIAT, leading to a high frequency of egg chambers with epithelial gaps (Figure 7F). Thus, increased apical contractility is sufficient to disrupt epithelial integrity in a proliferative epithelium, further supporting the idea that aPKC protects epithelial integrity through regulation of apical actomyosin.

\section{Discussion}

Apical-basal polarity provides positional information at the cellular level that is essential for tissue architecture. However, it remains ill-defined how loss of polarity regulators leads to epithelial architecture defects. Even though genetic approaches have yielded substantial insight, the inherent temporal constraints preclude direct visualization of the underlying events. Here, we used fast aPKC perturbation approaches in Drosophila epithelia to shed light on how aPKC regulates epithelial architecture. We show that epithelial gaps form prior to loss of apical-basal polarity and within minutes of aPKC downregulation in the follicular epithelium. aPKC inactivation increases apical contractility in non-mitotic cells. This increase pulls dividing and neighbor cells apart and ruptures the epithelium. Thus, we propose that aPKC downregulates apical contractility in polarized epithelia to prevent the build-up of excessive forces that compromise epithelial integrity (Figure 7G).

Rapid protein perturbation approaches are necessary to define the primary cellular cause of phenotypes in tissues. Here, we have developed a strategy to quickly inactivate aPKC in epithelial and neural stem cells by employing optogenetic clustering in the abdomen of living flies or ex vivo in intact organs. By complementing optogenetic clustering with the ability to adjust aPKC activity with chemical-genetics, we show that, immediately after clustering, aPKC is only partially inactive. More importantly, this allowed us to show that decreasing aPKC activity initially increases apical contractility and leads to the formation of gaps in the follicular epithelium. Strikingly, partial inactivation did not disrupt apical-basal polarity immediately, revealing that this is not the direct cause of epithelial gaps. This recapitulates the phenotype of hypomorphic aPKC alleles that produce gaps but do not disrupt apical-basal polarity $[11,45]$. Our results therefore indicate that a high threshold of aPKC inactivation is required to disrupt apical-basal polarity, which suggests that polarized epithelia can withstand fluctuations in aPKC activity. In turn, the higher sensitivity of the apical 
actomyosin cytoskeleton likely enables aPKC-dependent regulation of contractility without compromising apical-basal polarity.

aPKC is essential for apical-basal polarity, which provides spatial cues that orient the organization of the apical actomyosin network. However, aPKC has also been reported to antagonize the apical actomyosin network during morphogenesis in Drosophila and mammalian embryos $[35,56,57]$. We now propose that aPKC downregulates apical contractility to balance forces within proliferating epithelia to maintain epithelial integrity. Interestingly, whereas inhibition of aPKC induces accumulation of myosin at the apicomedial surface in follicle cells, myosin increase is more pronounced at junctions in neuroepithelial tissue. Consequently, our work highlights a primary role for aPKC as a negative regulator of apical constriction whose function can be locally regulated for different morphogenetic and homeostatic purposes. The molecular nature of this function has yet to be uncovered. Phosphorylation of ROCK by aPKC induces its cortical dissociation to downregulate junctional contractility in mammalian cells [26], but aPKC does not regulate equivalent sites in Drosophila Rok [58]. Alternatively, aPKC may target other actomyosin regulators or function through other apical polarity proteins implicated in the regulation of apical contractility, such as Crumbs and Lulu2/Yurt [34, 59-63].

Our findings suggest that physical constraints also define the phenotypic outcome of apical constriction. Our analysis shows that epithelial gaps form almost exclusively at the dorsal/ventral regions of egg chambers, where tension at the apical domain has been reported to be higher [54,64]. The follicular epithelium is physically constrained at the basal side by a stiff basement membrane [65] and at the apical side by the growing germline, which may keep the epithelium stretched $[66,67]$. Thus, egg chamber organization likely opposes the shape changes necessary to accommodate global apical constriction, leading to an increase in tension and rupture upon aPKC inactivation. In contrast, neuroepithelial cells constrict their apical area freely, which should release tension. Accordingly, while partial aPKC inactivation consistently led to large epithelial gaps in the follicular epithelium, in the neuroepithelium only minor perturbations at apical cell contacts were detected. Hence, on top of possible differences in the local response at the junctional or medioapical level, our findings stress the importance of physical boundaries, tissue geometry and mechanical context on the outcome of increased apical contractility.

We also provide direct evidence that large epithelial rupture can arise by intercellular detachment during cell division, which provides a weak spot primed for disruption upon increased mechanical stress. This is consistent with previous observations that reinforcement of junctional attachment to the cytoskeleton prevents detachment during cell division in the Drosophila embryonic epithelium and mammalian cell culture [68,69]. Dividing cells do not generate gaps upon aPKC inactivation in a mosaic tissue, showing that a direct effect in mitotic cells is not responsible for gap formation by itself. Then, why are mitotic cells prone to separate from hypercontractile surrounding tissue? Mitotic cells downregulate apicomedial actomyosin and revert apical constriction, which makes them more susceptible to extrinsic forces [70, 71]. We show that increased pulling forces exerted by the constricting non-mitotic tissue indeed expand the apical surface of mitotic cells upon aPKC clustering. These forces could amplify outward pulling forces at the poles of dividing cells [72], and spatially oppose pulling forces by the contractile ring on cell adhesion during cytokinesis. We observed that ruptures generally occur next to the equatorial region during cytokinesis, which is consistent with opposing forces overcoming cell adhesion in this region (Figure 7G). Furthermore, local remodelling of cell adhesion during mitosis $[39,73,74]$ and cytokinesis $[38,75-78]$ may favour detachment next to dividing cells. 
Different cellular events have to be integrated at the tissue level to drive concerted shape changes during morphogenesis. Apical constriction is frequently used to bend or fold epithelia during development [79]. Cell division actively contributes to tissue morphogenesis by controlling tissue material properties [74, 80-82] and driving shape change [83-85] or cellular rearrangements [86]. However, the cell-intrinsic mitotic remodelling of the cytoskeleton can disrupt morphogenetic processes that require apical constriction [70, 87-89]. Our results now show that forces produced by apical constriction challenge cohesion at the dividingneighboring cell interface, and thereby disrupt epithelial integrity. Hence, this study shows the importance of a strict control over apical constriction in proliferative tissues, so as to enable growth and morphogenesis without compromising epithelial integrity.

\section{Acknowledgments}

We thank Daniel St Johnston, Juergen A. Knoblich, Stefano de Renzis, Xiaobo Wang, Yohanns Bellaiche and the Bloomington Drosophila Stock Center for fly stocks. We also thank Yohanns Bellaiche, Ivo Telley and Romain Levayer for insightful comments on the manuscript. This work was funded by National Funds through FCT-Fundação para a Ciência e a Tecnologia, I.P., under the projects PTDC/BEX-BCM/0432/2014 and PTDC/BIACEL/1511/2021. E.M. is funded by "FCT Scientific Employment Stimulus - Individual Call" program (CEECIND/00622/2017). M.O. and A.M.C. were supported by PhD fellowships from Bioimaging (PPBI-POCl-01-0145-FEDER-022122). Work in JJ's lab is supported by the $100031 / Z / 12 / Z$ and 100031/Z/12/A from Wellcome and the Royal Society. We would like to thank the Dundee Imaging Facility for excellent support.

\section{Author contributions}

405

E.M. and M.O. conceptualized the study and wrote the original manuscript draft; Data acquisition: M.O., A.B., A.M.C. and E.M. performed all experiments in follicular epithelium apart of the experiment performed by N.L. in Fig. 6D; N.L performed experiments in larval neuroepithelium; P.G. performed neuroblast experiments. Data analysis and interpretation: M.O., A.B., A.M.C., N.L., P.G., C.H, J.J., and E.M.; Supervision: C.S., C.H., J.J. and E.M. Funding acquisition: C.H, J.J. and E.M.; All authors reviewed the manuscript.

\section{Declaration of interests}

412 The authors declare no competing interests. 

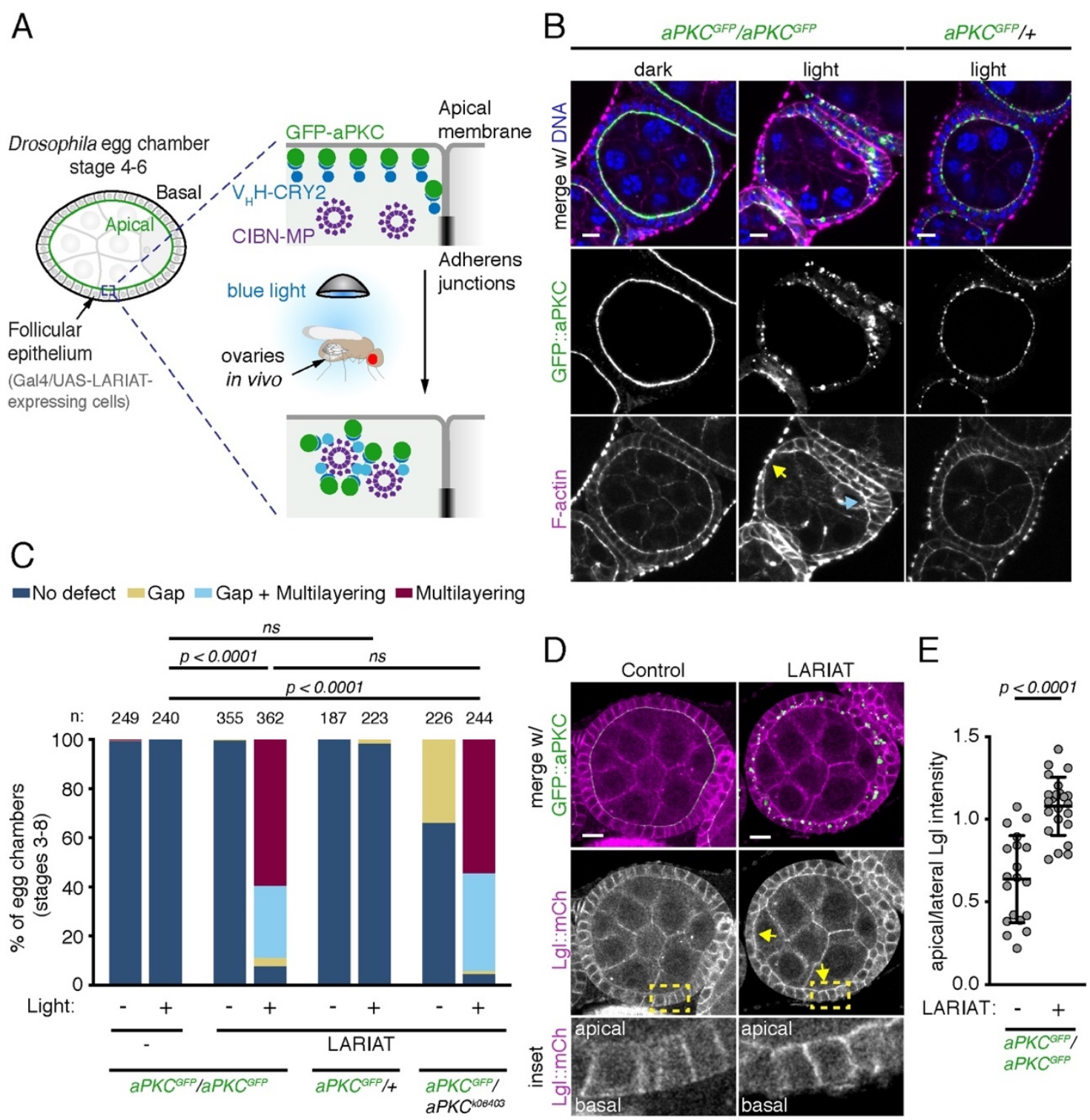

Figure 1. Optogenetic clustering inactivates aPKC and disrupts tissue architecture in vivo.

(A) Schematic representation of optogenetic aPKC inactivation strategy using LARIAT ( $\mathrm{V}_{\mathrm{H} H} \mathrm{H}: \mathrm{CRY} 2$ and CIBN::MP). GFP::aPKC is targeted by CRY2 fused with a GFP nanobody $\left(V_{H} H\right)$. Exposing flies to blue light triggers $C R Y 2$ binding to CIBN fused with a multimerization domain (MP) and clusters GFP::aPKC. (B) Living flies were exposed to blue light for 48 hours to cluster GFP::aPKC or kept in the dark (control) before egg chambers were stained for F-actin and DNA. Control flies were kept in the dark. Flies were either homozygous or heterozygous for endogenously-tagged GFP::aPKC. Arrows point to epithelial gap (yellow) and multilayering (cyan). (C) Frequency of epithelial defects in egg chambers (stages 3 to 8) from flies with the indicated combinations of wild-type, GFP::aPKC or apkck06403 null allele after 24 hours blue light exposure ( $n=$ number of egg chambers). LARIAT was expressed in the follicular epithelium when indicated. Control flies were kept in the dark. Fisher's exact test compared the incidence of defects between different samples (ns, not significant). (D) Representative midsagittal images of control and LARIAT egg chambers from flies expressing GFP::aPKC and Lgl::mCherry exposed to blue light for 24 hours. Arrows point to apical Lgl::mCherry. Yellow boxes define region in insets. (E) Ratio of apical/lateral mean pixel intensity of Lgl::mCherry in control $(n=684$ cells, 19 egg chambers) and LARIAT ( $n=447$ cells, 23 egg chambers). Graphs show mean \pm SD, grey points represent average for individual egg chambers (t-test). Scale bars: $10 \mu \mathrm{m}$. See also Figure $\mathrm{S} 1$. 

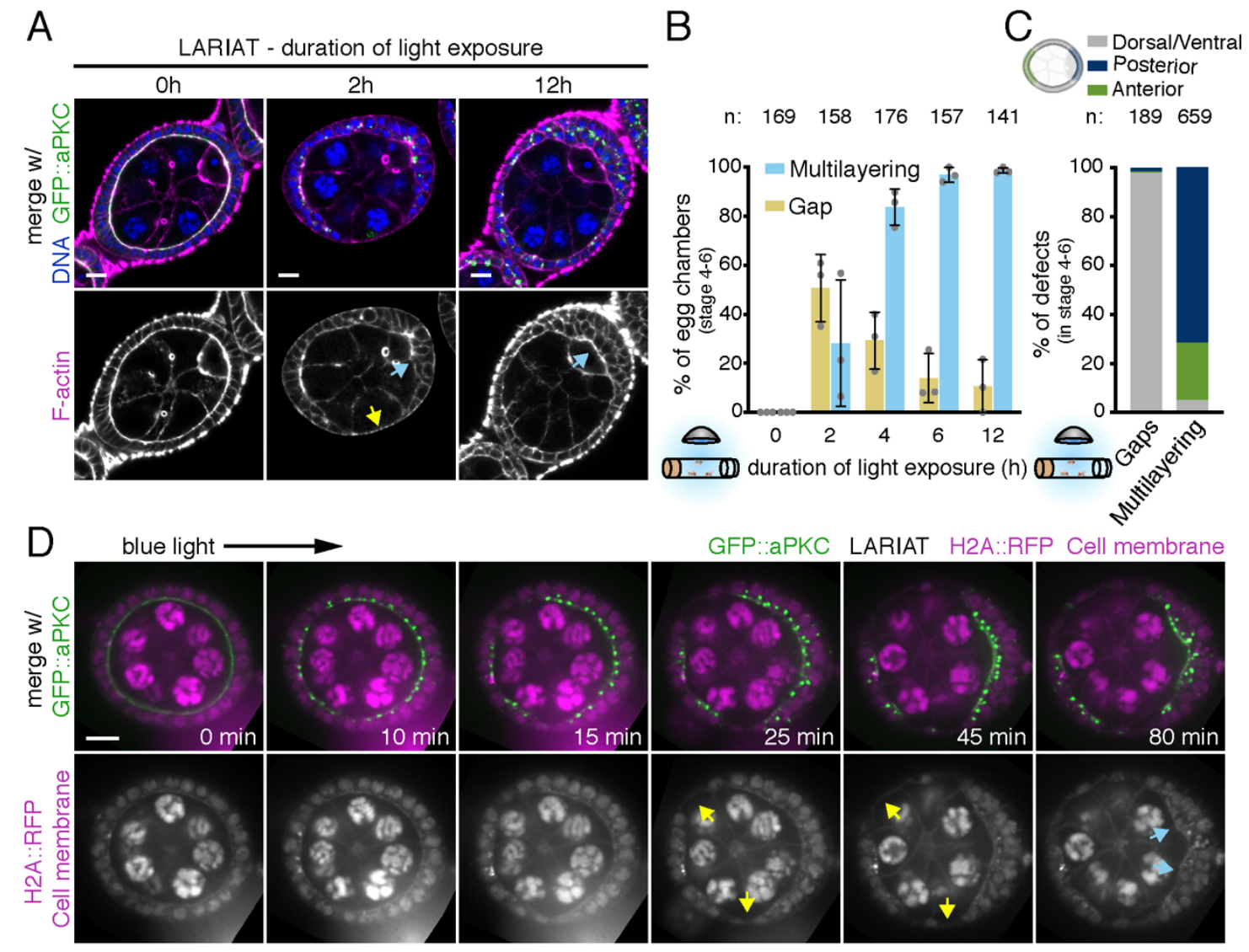

$\mathrm{E}$

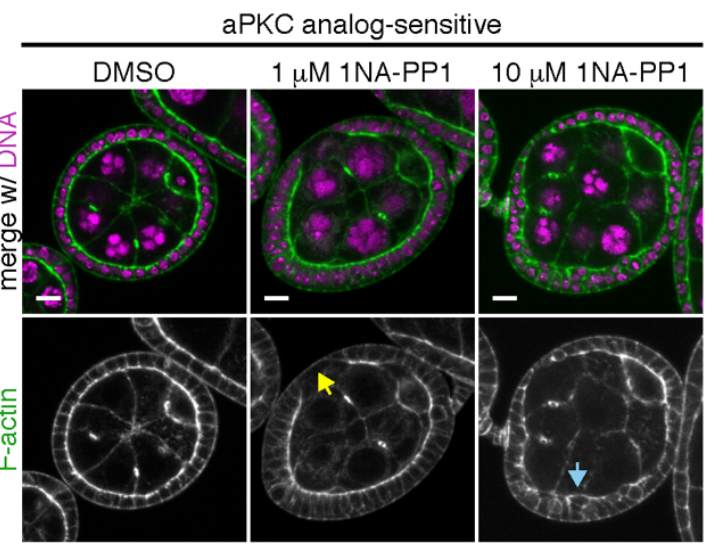

$\mathrm{F}$

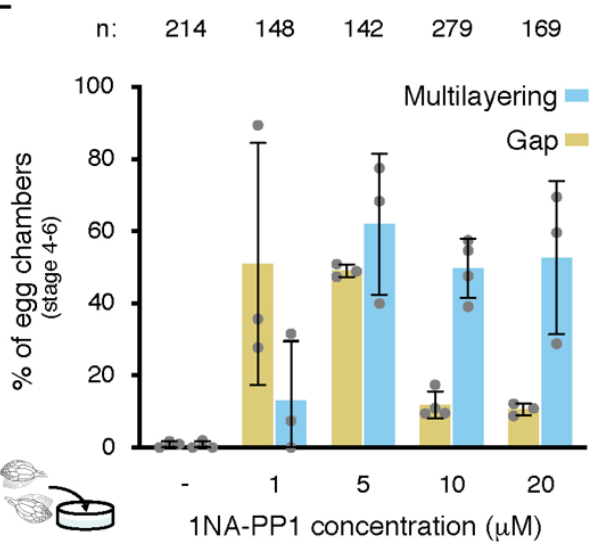

Figure 2. aPKC inactivation leads to fast tissue disorganization in a proliferative epithelium.

(A-C) Representative midsagittal images and quantification of epithelial defects in GFP::aPKC homozygous egg chambers in proliferative stages (4-6) expressing LARIAT and stained for F-actin and DNA. Flies were exposed to light for the indicated time before ovary fixation. (B) Frequency (mean \pm SD) of epithelial gaps (yellow arrow in A) and multilayering (cyan arrows in A). (C) The data from (B) was re-analyzed to show the spatial distribution of all defects, $n=$ total amount of gaps or multilayering events. (D,E) Time-lapse midsagittal images of an egg chamber expressing LARIAT (in the follicular epithelium), GFP::aPKC and H2A::RFP show epithelial gaps (yellow arrows) and multilayering (cyan arrows). Imaging with 488nm laser triggered LARIAT clustering from min 0 onwards. (E,F) Midsagittal images and epithelial defect quantification in proliferative $a P K C^{\text {as } 4}$ egg chambers treated ex vivo for 2 hours with the indicated concentrations of 1NA-PP1 to inactivate aPKC and stained for F-actin and DNA. (F) Graph shows frequency (mean \pm SD) of epithelial gaps (yellow arrow) and multilayering (cyan arrow). Grey data points (graphs in (B,F)) represent independent experiments; $n=$ number of egg chambers. Scale bars: $10 \mu \mathrm{m}$. 
bioRxiv preprint doi: https://doi.org/10.1101/2022.03.02.482459; this version posted March 4, 2022. The copyright holder for this preprint (which was not certified by peer review) is the author/funder, who has granted bioRxiv a license to display the preprint in perpetuity. It is made available under aCC-BY-NC-ND 4.0 International license.
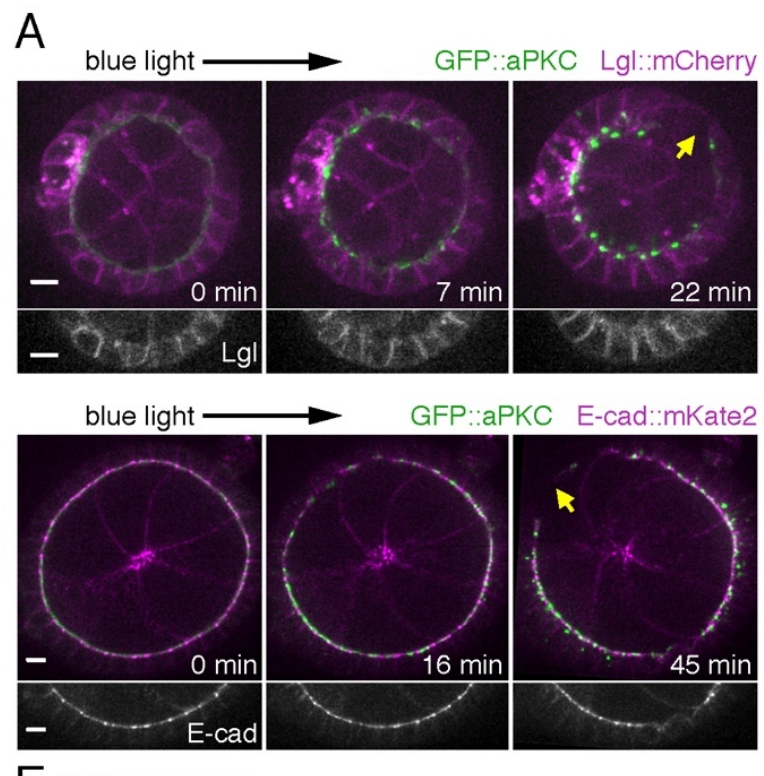

E cell membrane
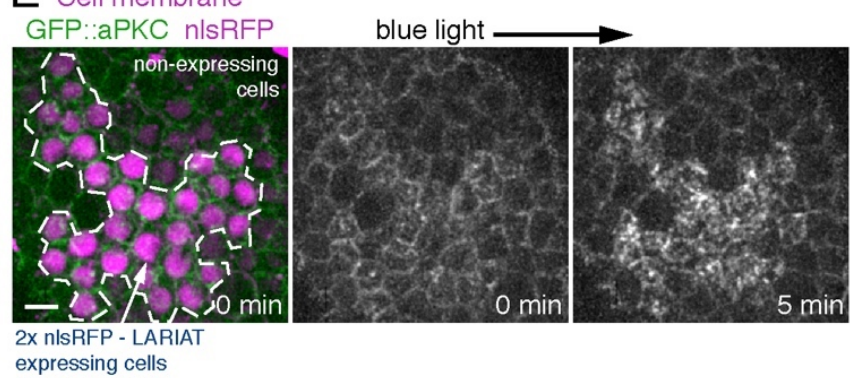

$0 \mathrm{~min}$

expressing cells
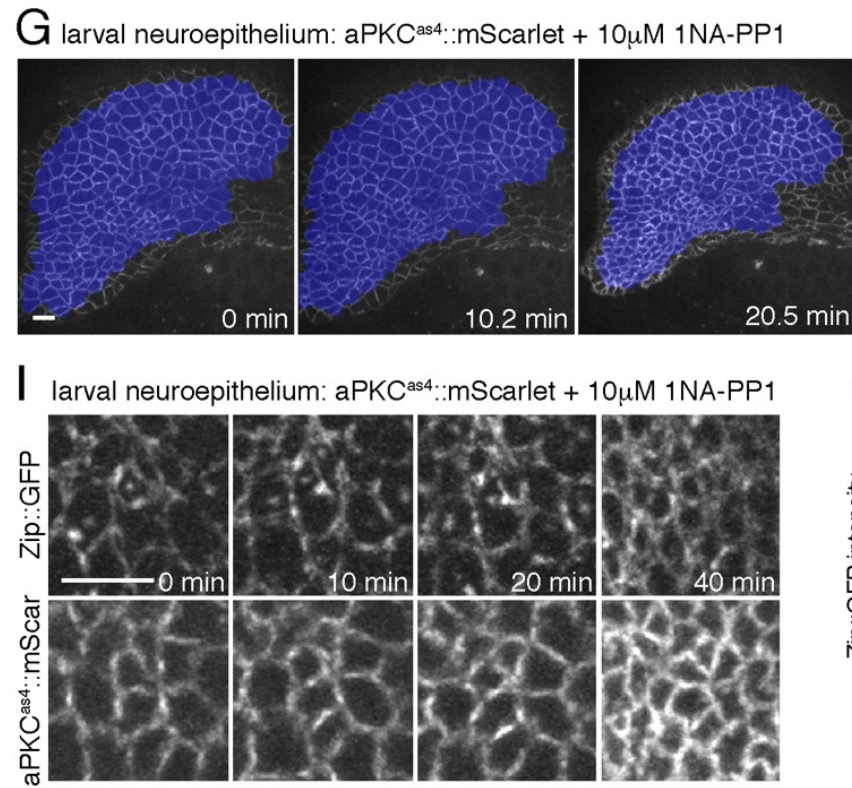
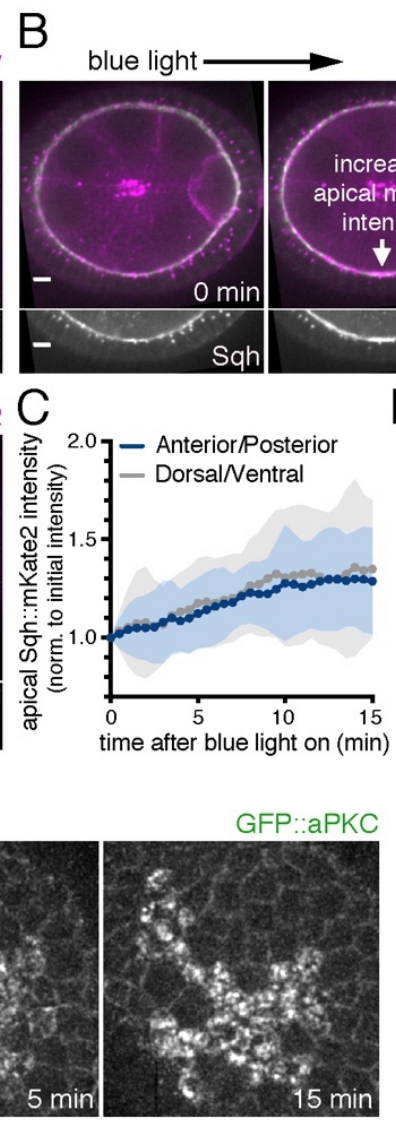

$\mathrm{F}$
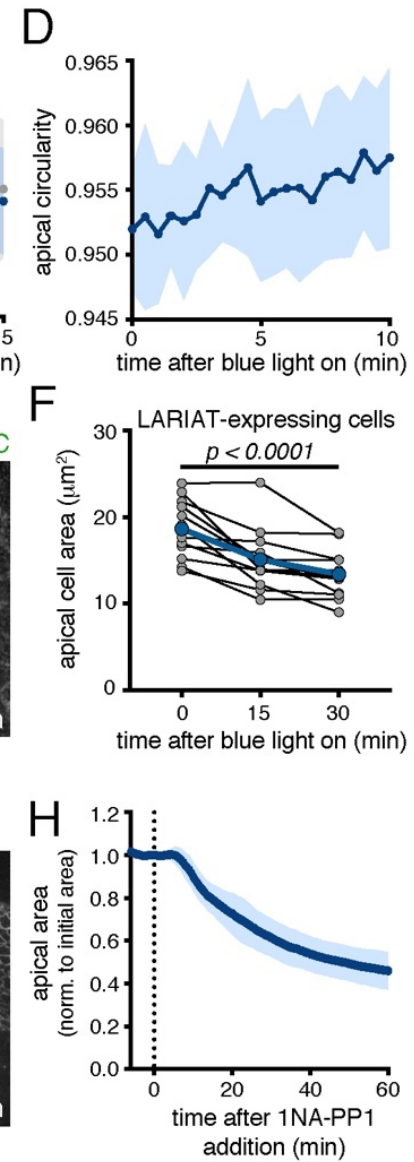

Figure 3. aPKC antagonizes apical constriction in Drosophila tissues

(A, B) Time-lapse midsagittal images of egg chambers expressing LARIAT, GFP::aPKC and either (A top) Lgl-mCherry, (A bottom) E-cad::mKate2 or (B) Sqh::mKate2. Imaging with 488 $\mathrm{nm}$ laser triggered aPKC clustering from min 0 onwards. Yellow arrows in (A) indicate epithelial gaps. (C) Sqh accumulation at the apical surface after aPKC clustering (mean \pm SD) measured at the anterior/posterior (AP) and dorsal/ventral (DV) regions, corrected for cytoplasm intensity and normalized to its initial value ( $n \geq 96$ AP and $\geq 96$ DV cells, 12 egg chambers). (D) Egg chamber circularity (mean \pm SD) was measured at the apical surface of the follicular epithelium 
( $n=10$ egg chambers). (E) Time-lapse images of GFP::aPKC follicular epithelium cells with mosaic LARIAT expression (marked by $2 x n l s:: R F P$ in magenta). Imaging with $488 \mathrm{~nm}$ laser triggered aPKC clustering and apical domain constriction in LARIAT-expressing cells. (F) Average apical cell area (mean \pm SD) within LARIAT expressing clones before and after aPKC clustering. Points represent average for individual clones $(n=161$ cells, 12 clones, $p<0001$, ANOVA for paired samples). (G) Contraction of aPKC ${ }^{\text {as } 4:: m S c a r l e t ~ l a r v a l ~ b r a i n ~ n e u r o e p i t h e l i u m ~}$ following the addition of $10 \mu \mathrm{M} 1 \mathrm{NA}-\mathrm{PP} 1$ at min 0 . (H) Graph shows apical area (mean \pm SD, normalized to its initial value) of aPKCas4::mScarlet larval brain neuroepithelial tissue after addition of $10 \mu \mathrm{M}$ 1NA-PP1 ( $\mathrm{n}=10$ neuroepithelia with an average of 157 cells, 5 independent experiments). (I) Close-up of the neuroepithelium of an $a P K C^{a s} 4:: m S c a r l e t$ larvae expressing nonmuscle myosin II heavy chain (Zip::YFP) following addition of $10 \mu \mathrm{M}$ 1NA-PP1. (J) Zip::YFP intensity (mean \pm SD) at the apical junctions and medioapical region (quantification masks shown) was normalized to junction intensity at $-2 \min (n=4$ neuroepithelia with $\geq 108$ cells). 


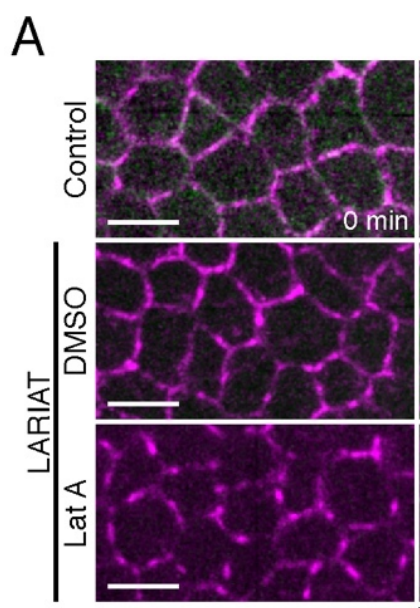

Before blue light exposure

C

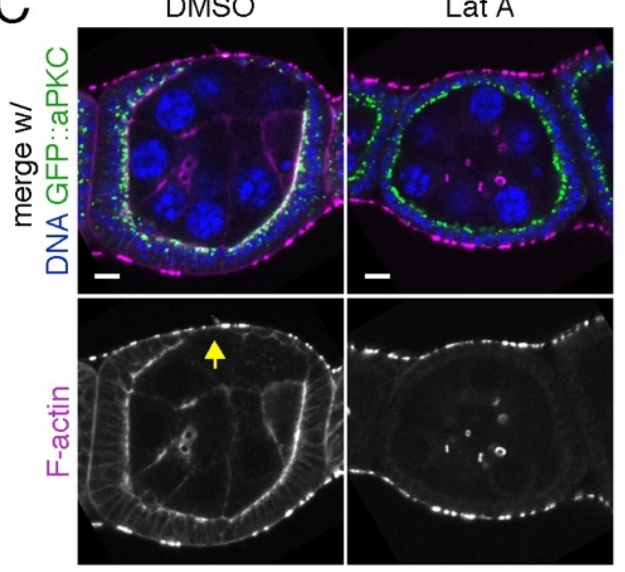

GFP::aPKC E-cad::mKate2
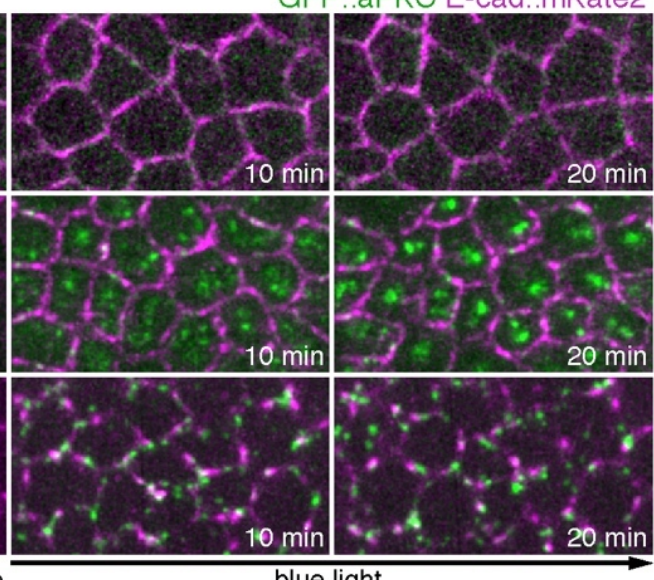

blue light

D n: 252207143256

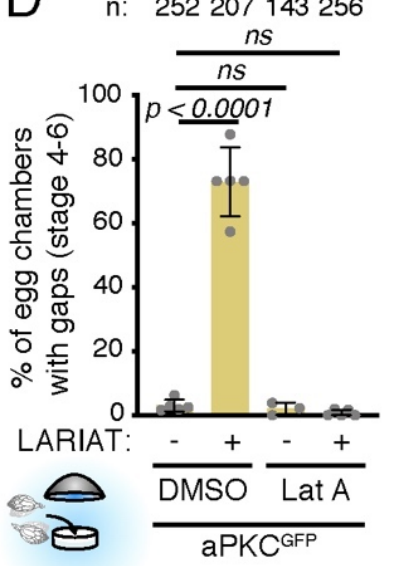

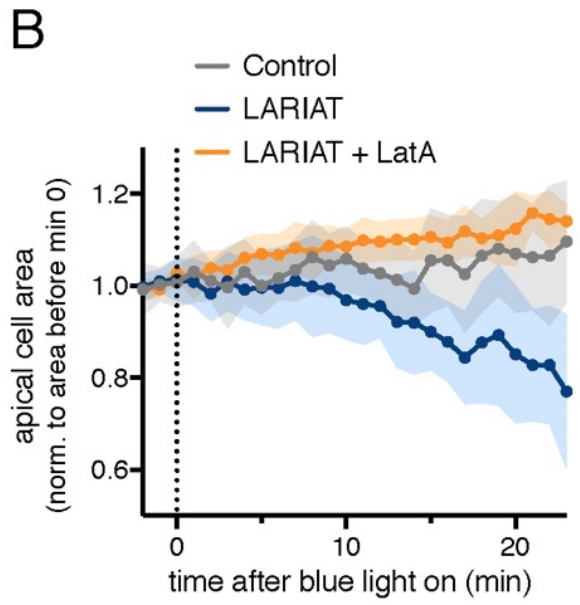

$E$ n: $123|121142| 125121|112103| 115$

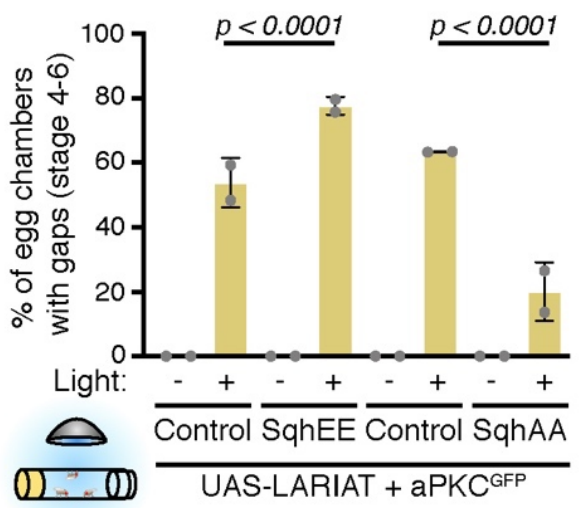

Figure 4. Increased apical contractility underlies gap formation after aPKC optogenetic 516 clustering.

517 (A) Live imaging of GFP::aPKC in control or LARIAT egg chambers co-expressing E-cad::mKate2 518 (apical view) and treated or not with Lat A prior to aPKC clustering (bottom). Imaging with the $488 \mathrm{~nm}$ 519 laser triggered aPKC clustering from min 0 onwards. (B) Apical surface area (mean \pm SD) measured at the junction level and normalized to the mean value before clustering ( $n \geq 7$ egg chambers per condition). (C,D) Representative midsagittal images and quantification of epithelial gaps in proliferative follicular epithelium expressing GFP::aPKC (green) and LARIAT and stained for F-actin and DNA. Ovaries were exposed to blue light ex vivo before fixation. Actin disruption by Lat $A$ treatment restores epithelial integrity. (D) Frequency (mean $\pm S D$ ) of epithelial gaps (yellow arrow in C) scored in the presence (+) or absence of LARIAT (-) in egg chambers treated with DMSO (control) or Lat A. (E) Epithelial gap frequency (mean $\pm S D$ ) was scored upon overexpression of mCherry (Control), Sqh ${ }^{\text {E20E21 }}$ (SqhEE) or Sqh ${ }^{\text {A20A21 }}$ (SqhAA) in the follicular epithelium of GFP::aPKC LARIAT flies. Flies were exposed to blue light (+) or kept in the dark (-) for 2 hours prior to fixation; grey data points represent 
A

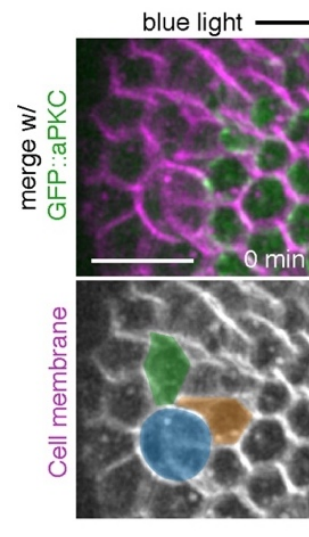

C

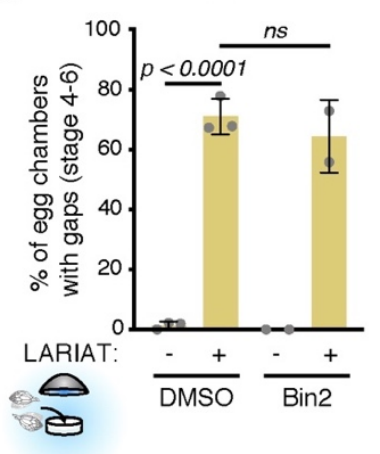

GFP*.aPKC Cell membrane

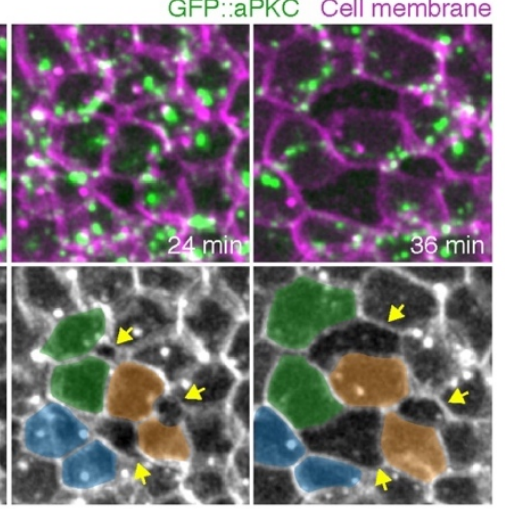

$\begin{array}{llllll}\mathrm{D} & \mathrm{n}: & 113 & 81 & 128 & 115\end{array}$

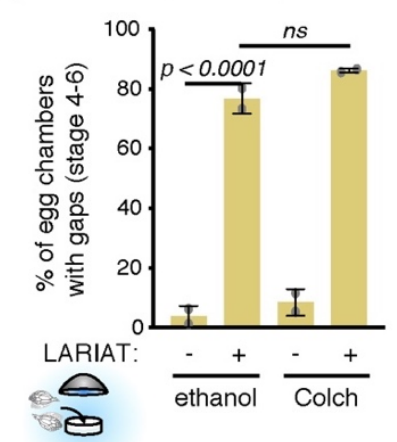

$\mathrm{E}$
B interphase

mitosis

cytokinesis

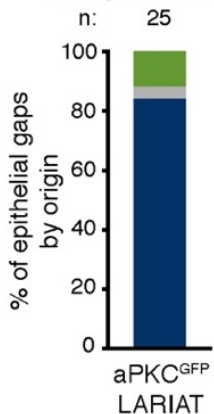

$\mathrm{F}$
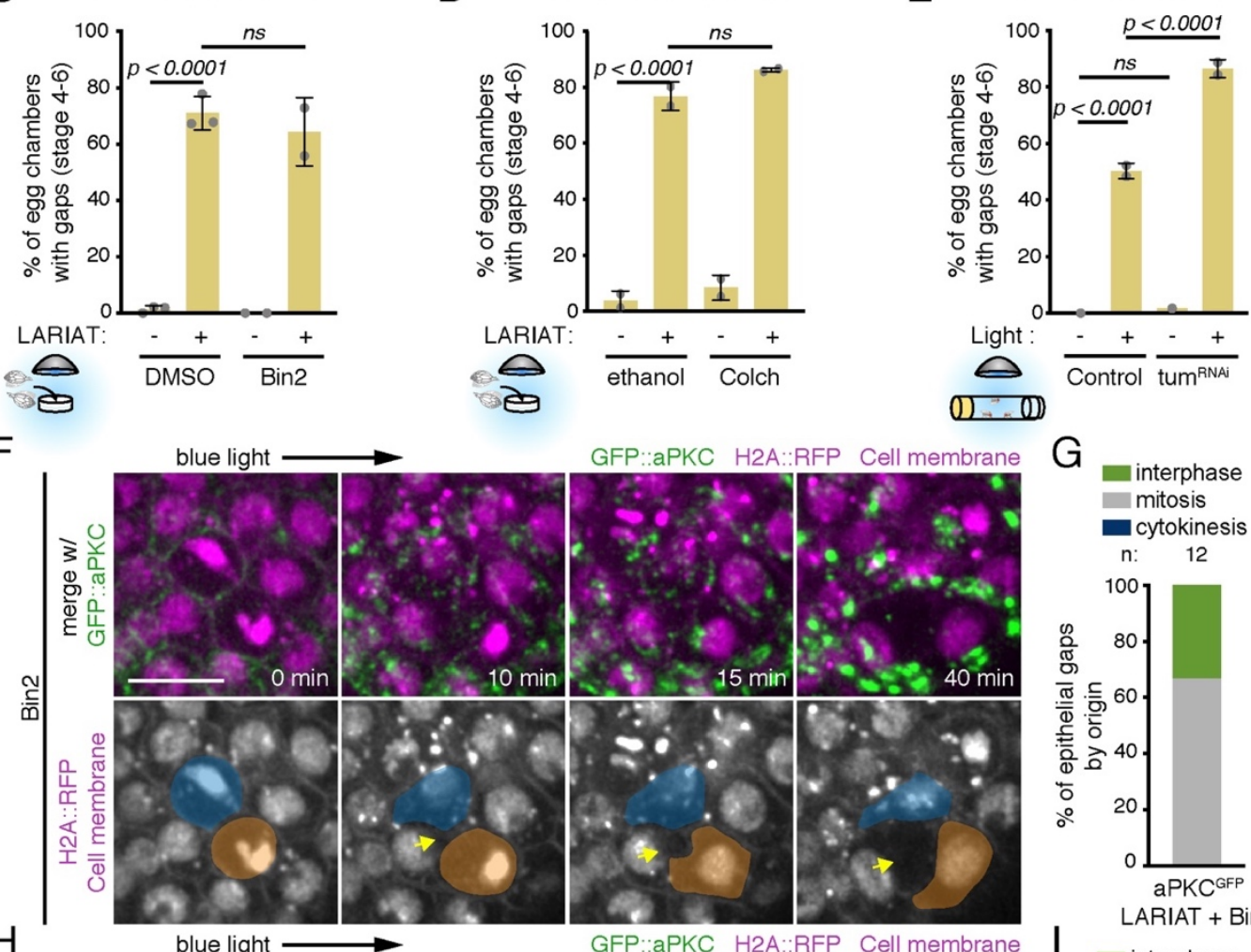

G

interphase mitosis cytokinesis

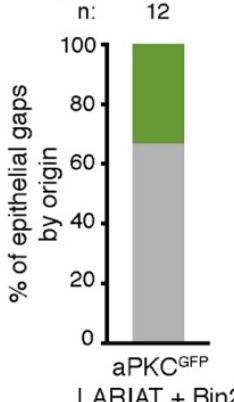

$\mathrm{H}$

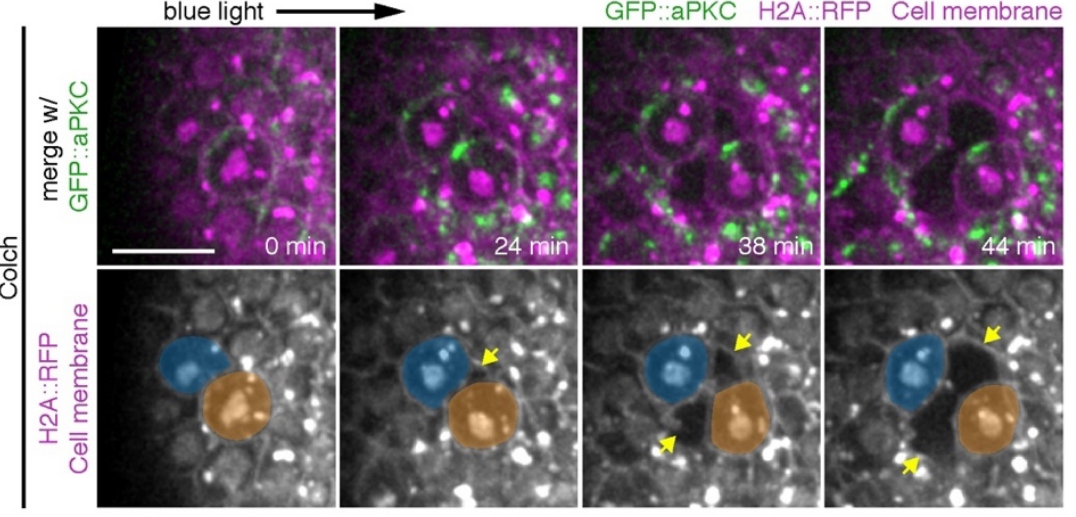

I

$$
\text { LARIAT + Bin2 }
$$

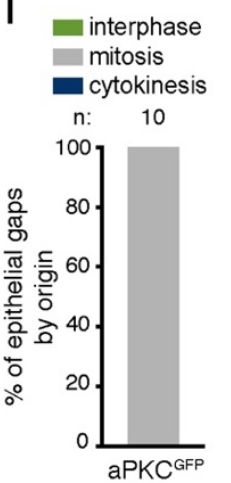

LARIAT + Colch

539 Figure 5. Cell division challenges tissue cohesion upon aPKC inactivation.

540 (A) Time-lapse images of an egg chamber (surface view) expressing LARIAT, GFP::aPKC and stained 541 with membrane marker. Imaging with 488nm laser clustered aPKC from min 0 onwards. Epithelial gaps 542 (arrow) form adjacent to dividing cells (colored). (B) Quantification of epithelial gap origin according to 543 cell division stage of neighbouring cells. $n=$ number of gaps scored. (C,D) Gap frequency in the 
presence and absence of LARIAT in egg chambers treated with the indicated drug: (C) Binucleine-2 (Bin2), to inhibit AurB, or (D) Colchicine (Colch), to depolymerize microtubules, before light exposure for 2 hours ex vivo. (E) Frequency of epithelial gaps scored in control and Tum RNAi egg chambers from flies expressing GFP::aPKC LARIAT and exposed (+) or not (-) to blue light for 2 hours. Graphs in (C-E) show mean \pm SD; grey data points represent independent experiments; $n=$ number of egg chambers scored; Fisher's exact test was used (ns, not significant). (F-I) Time-lapse images of follicular epithelium (surface views) expressing LARIAT, GFP::aPKC and H2A::RFP and stained with membrane marker. (F) Bin-2 or (H) Colch were added at least 15 min prior to clustering from min 0 onwards. Epithelial gaps (arrows) form adjacent to dividing cells (colored) despite $(\mathbf{F})$ cytokinesis failure (chromatin (H2A::RFP) decondenses without chromosome separation) or $(\mathbf{H})$ mitotic arrest (condensed chromatin throughout the movie). (G,I) Epithelial gap origin according to cell division stage of neighbouring cells. $n=$ number of gaps scored. Scale bars: $10 \mu \mathrm{m}$. See also Figure S3. 

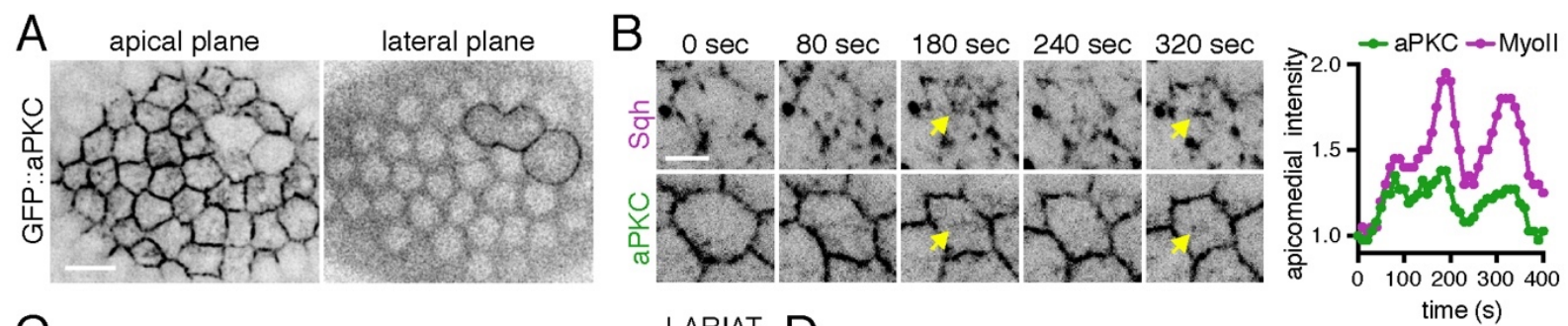

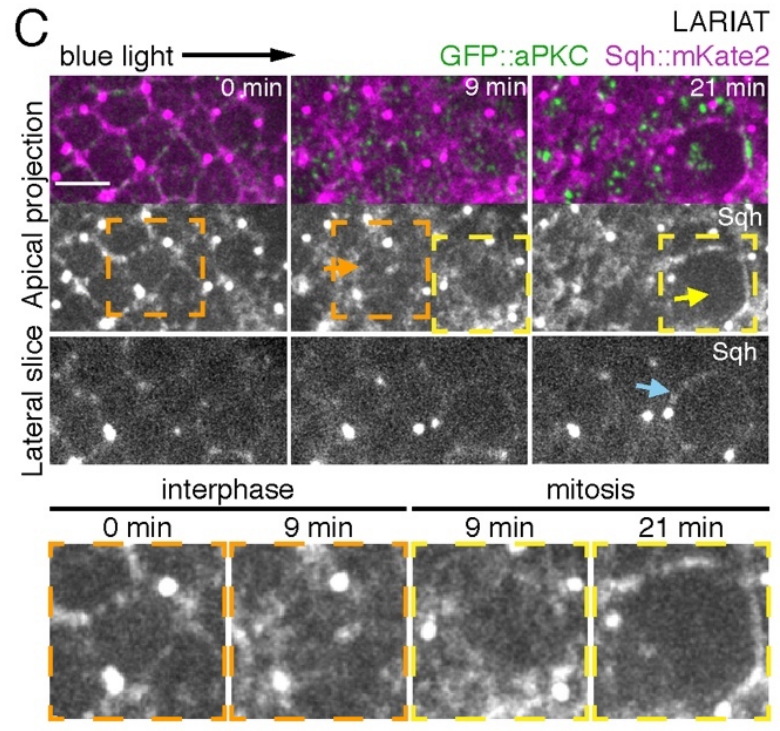

E

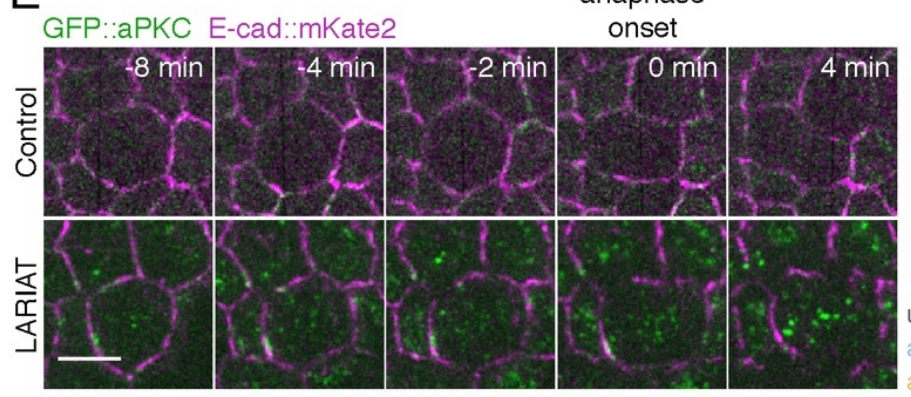

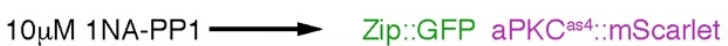

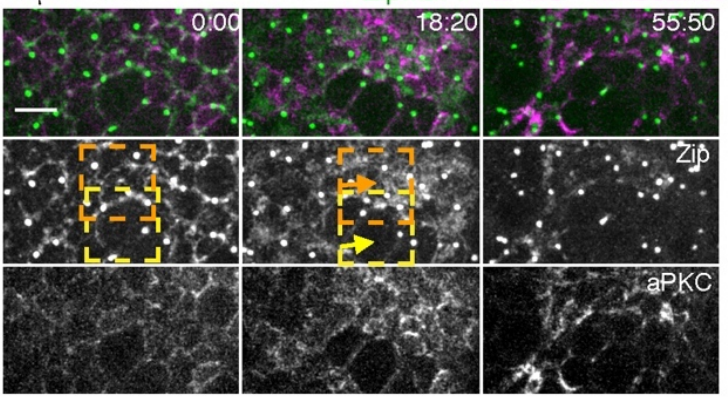

mitosis

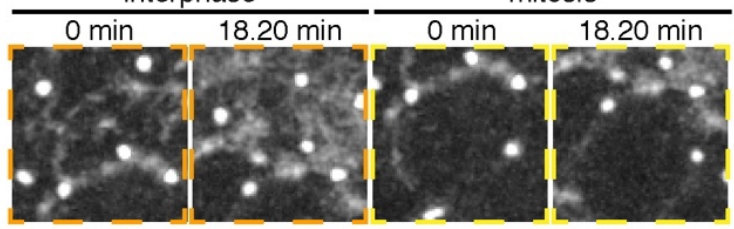

F

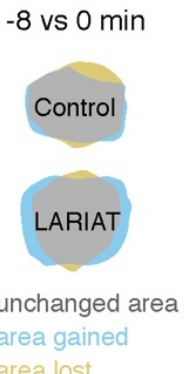

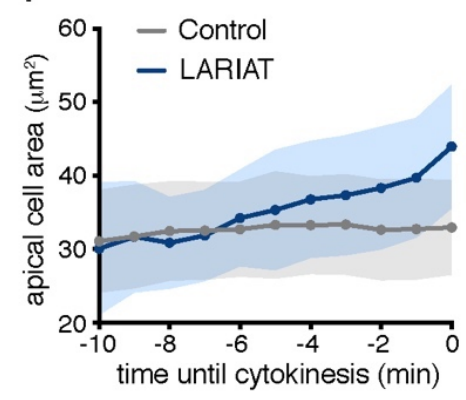

596

597

598

599

600

601

602

603

604

605

606

607

608

609

610

611

612

613

614

Figure 6. aPKC inactivation leads to excessive pulling forces on dividing cells.

(A) GFP::aPKC in the follicular epithelium at the apical surface (left) and at the lateral cortex (right). (B) Live-imaging of GFP:::aPKC and Sqh::mKate2 at the apical surface shows dynamic accumulation at the apicomedial region (arrows). Normalized, background subtracted mean pixel intensity of GFP::aPKC and Sqh::mKate2 measured in the medioapical region using a circular $2.5 \mu \mathrm{m}$ diameter ROI (right). (C) Live imaging of GFP::aPKC LARIAT in the follicular epithelium with Sqh::mKate2. Imaging with the 488 $\mathrm{nm}$ laser triggered aPKC clustering from min 0. After GFP::aPKC clustering, Sqh::mKate2 accumulates at the apicomedial region in interphase cells (orange arrow), but not in mitotic cells (yellow arrows), where aPKC clustering does not affect redistribution to the lateral cortex (blue arrow). (D) Time-lapse images of $a P K C^{a 4} 4:: m S c a r l e t$ (magenta), Zip::YFP (green) follicle cells treated with $10 \mu \mathrm{M} 1 \mathrm{NA}-\mathrm{PP} 1$ at timepoint 0 . Transient apicomedial accumulation of Zip::YFP (orange arrow) is not observed in mitotic cells (yellow arrow). (C,D) Insets show individual interphase and mitotic cells. (E) Live imaging of GFP::aPKC and E-cad::mKate2 in the follicular epithelium (apical projection). aPKC clustering was triggered up to $5 \mathrm{~min}$ before mitotic entry. Cells enter anaphase at min 0 . (F) Apical surface area in dividing cells was measured at the junction level until anaphase onset $(n=27$ cells from 6 control egg chambers and 12 cells from 8 LARIAT egg chambers. Graphs show mean \pm SD. Scale bars: $5 \mu \mathrm{m}$. 
A

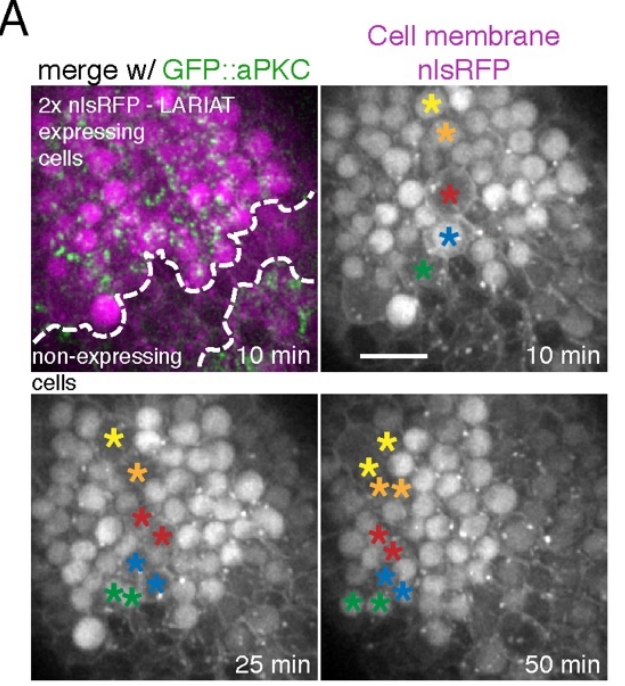

B

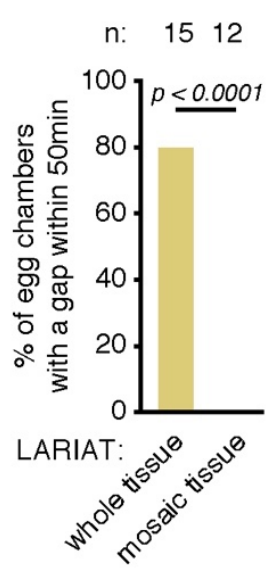

C

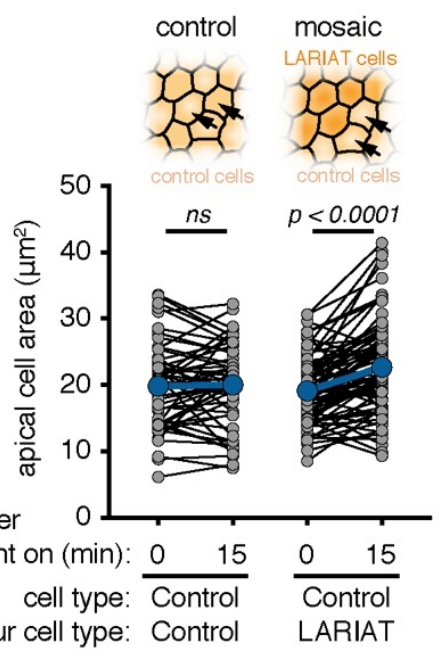

time after

blue light on (min): $\frac{0 \quad 15}{\text { Control }} \quad \frac{0 \quad 15}{\text { Control }}$

neighbour cell type: Control LARIAT

D

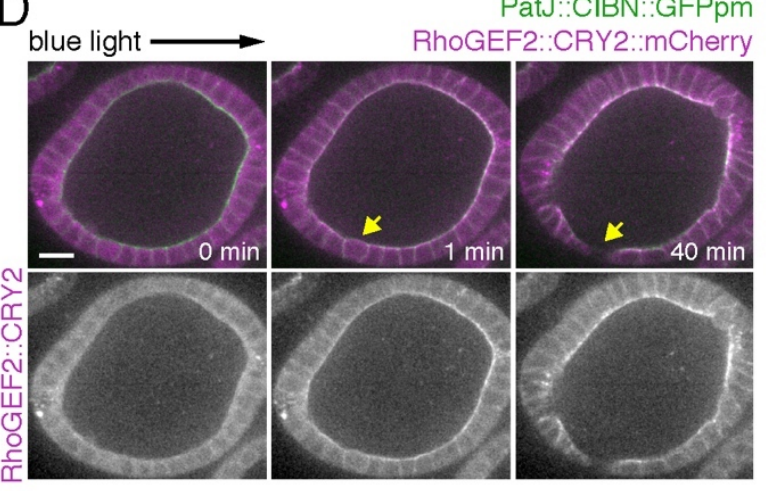

$\mathrm{E}_{\text {blue light } \longrightarrow}$

PatJ::CIBN::GFPpm
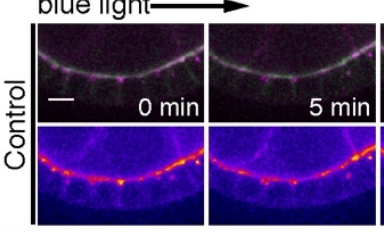

Sqh:mKate?
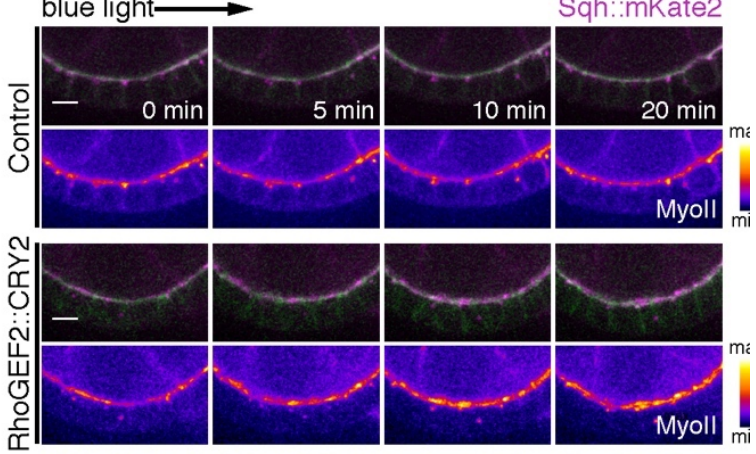

F
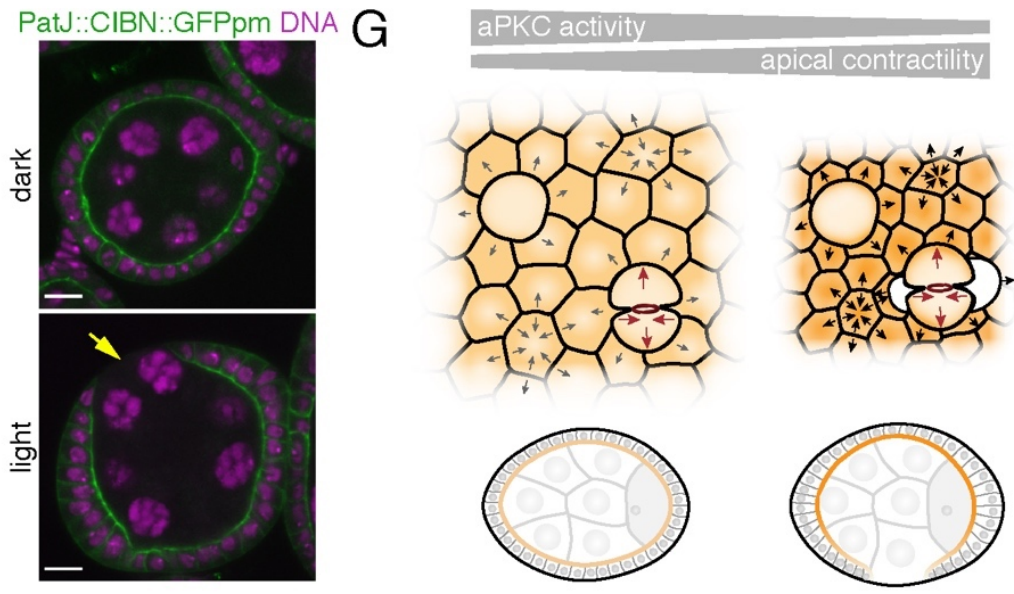

Figure 7. Global increase of apical contractility induces epithelial gaps.

(A) Time-lapse images (surface of egg chamber) show epithelial rupture does not occur in GFP::aPKC follicular epithelia with mosaic LARIAT expression (marked by nls::RFP in magenta) despite multiple dividing cells (asterisks). (B) Frequency of gaps in tissue with mosaic LARIAT expression compared with whole tissue expression of LARIAT (data from Figure 5B re-analysed, Fisher's test). (C) Variation off apical cell area shows that wild-type neighbour cells (adjacent to LARIAT-expressing clones, $n=82$ cells, 13 egg chambers) expand within 15 min of aPKC clustering, unlike wild-type cells in a fully wildtype tissue ( $n=60$ cells, 2 egg chambers). Points represent individual cells (ANOVA for paired samples). (D) Live imaging of egg chamber expressing PatJ::CIBN::pmGFP and RhoGEF2::CRY2::mCherry in the follicular epithelium (midsagittal view). Imaging with $488 \mathrm{~nm}$ laser targeted RhoGEF2 to the apical domain from timepoint 0 onwards. Yellow arrow denotes gap formation in a region with a dividing cell. (E) Time-lapse images of the follicular epithelium expressing 
PatJ::CIBN::pmGFP, RhoGEF2::CRY2 and Sqh::mKate2 (midsagittal view). Pseudo-colored Representative midsagittal images and epithelial gap (yellow arrow) quantification in egg chambers (stages 4 to 6) from flies expressing PatJ::CIBN::pmGFP and RhoGEF2::CRY2::mCherry exposed to blue light for 2 hours prior to fixation and staining for DNA. Control flies were left in the dark. Graphs show mean \pm SD; grey data points represent independent experiments; $n=$ total amount of egg chambers; Fisher's exact test was used (ns, not significant); Scale bars: $5 \mu \mathrm{m}$. (G) Model depicts how downregulation of aPKC activity increases apical constriction in the non-mitotic cells of a polarized epithelium, generating excessive pulling forces on dividing cells that induce detachment and, ultimately,

637 epithelial gaps.

638

639

640 
673

674

675

676

677

678

679

680

681

682

683

684

685

686

687

688

689

690

691

692

693

694

695

696

697

698

699

700

701

702

703

704

705

706

707

708

709

710

711

712

713

714

715

716

717

718

719

\section{METHODS}

\section{EXPERIMENTAL MODEL AND SUBJECT DETAILS}

\section{Drosophila melanogaster genetics and husbandry}

We performed all experiments using Drosophila melanogaster. We raised fly lines on standard fly food (cornmeal/agar/molasses/yeast) at $18^{\circ} \mathrm{C}$ or $25^{\circ} \mathrm{C}$ with $60 \%$ humidity and $12 \mathrm{~h} / 12 \mathrm{~h}$ dark light cycle, except when otherwise indicated in the method details section.

The following fly lines were used:

- Under regulation of the respective endogenous promoters: Par-6::GFP ([90], gift from Jürgen Knoblich, IMBA, Vienna), H2A::RFP ([91] and Sqh::mKate2x3 - Drosophila non-muscle myosin II regulatory light chain tagged with three tandem $m K$ ate2 and inserted in chromosomes II and III ([92], gift from Yohanns Bellaïche, Institut Curie, Paris);

- Tagged in the respective endogenous locus: GFP::aPKC ([43], gift from Daniel St Johnston, The Gurdon Institute, Cambridge), Lgl::mCherry [22] and E-cad::GFP [93], both gifts from Yang Hong, University of Pittsburgh), E-cad::mKate2x3 and Ecad::GFP 3 (tagged with three tandem mKate2 or GFP, [92], gift from Yohanns Bellaïche, Institut Curie, Paris) and Zip::YFP - Drosophila non-muscle myosin II heavy chain [94];

- UAS-LARIAT inserted in chromosomes II and III: CRY2 PHR domain fused to SNAPtag and a GFP nanobody $\left(V_{H} H\right)$ and $\mathrm{N}$-terminal $C / B$ domain (residues 1-170) fused to the CaMKIl $\alpha$ multimerization domain (MP), both expressed from a single construct with the help of a P2A self-cleaving peptide ([44], gift from Xiabo Wang, CBI, Université de Toulouse);

- For optogenetic RhoGEF2 recruitment to the apical membrane: PatJ::CIBN::pmGFP, for UAS-driven expression of PatJ fused to N-terminal CIB domain (residues 1-170) and membrane targeted GFP fused to human Kras4B CAAX [55], RhoGEF2::CRY2 and RhoGEF2::CRY2::mCherry, for UAS-driven expression of tagged and untagged catalytic DHPH domain of RhoGEF2 fused to CRY2 PHR [95], gifts from Stefano de Renzis, EMBL, Heidelberg);

- To drive Gal4-UAS mediated construct expression: GR1-Gal4, an enhancer trap line where Gal4 is under regulation of unknown regulatory sequences that drive Gal4 expression in the follicular epithelium ([96] and $t j-G a / 4$, an enhancer trap line where Gal4 is under regulation of traffic jam regulatory sequences (DGGR_104055); pnt-Gal4 (VDRC_VT212057, discarded)

- Gal8ts, temperature-sensitive Gal80 under regulation of the $\alpha$ Tub84B promotor (BDSC_7018);

- nlsRFP hs-Flp FRT19A (BDSC_31418) and hs-Flp Gal80 FRT19A (BDSC_5133), for FLP/FRT-mediated generation of Gal80 clones.

- $\quad a P K C^{K 06403}$, an $a P K C$ null allele obtained by insertional mutagenesis of a P-element construct [6];

- $a P K C^{a s 4}$ is an ATP analog-sensitive aPKC allele (I342A and T405A mutations introduced in the endogenous locus through CRISPR/Cas9 [48];

- aPKC::mScarlet ${ }^{\mathrm{as} 4}$ was made by scar-less (inDroso co-CRISPR approach) CrispR gene editing. The $\mathrm{mScarlet-l}$ sequence, preceded and followed by short two amino acid (VAL GLY) linkers, was inserted into the genome of the apkc $c^{\text {as4 }}$ line using the 
AATGGATCCTCCGGTGGCGGTGG guide RNA. The insert position was the same as previously published for GFP [97]: the mScarlet-I amino acid sequence, including the ATG and framed by the linkers, was inserted after amino acid 228 of aPKC-PA.

- UAS-mCherry (BDSC_35787), UAS-Tum RNAi (BDSC_28982) and phosphomimetic and nonphosphorylatable Sqh - UAS-Sqh ${ }^{\mathrm{E} 20 \mathrm{E} 21}$ (BDSC_64411) and UAS-Sqh ${ }^{\mathrm{A} 20 \mathrm{~A} 21}$ (BDSC_64114);

Fly genotypes for each experiment can be found in Table S1. For optogenetic experiments where flies were exposed to blue light, female offspring of the same cross with the same genotype were randomly assigned to experimental groups (dark vs light). For each independent ex vivo experiment with drug treatment of egg chambers, we dissected ovaries from all flies of the same genotype, mixed them together, separated their ovarioles and then randomly distributed them by the experimental groups. For live imaging of egg chambers, we imaged 2-3 egg chambers per fly.

\section{Optogenetic experiments in the follicular epithelium}

To inactivate apical polarity with optogenetics, we combined aPKC tagged with GFP in its endogenous locus [43] with UAS-LARIAT [44]. We used tj-Gal4 or GR1-Gal4 to drive UAS constructs expression in the follicular epithelium. To minimize premature UAS construct expression, crosses were kept at $18^{\circ} \mathrm{C} \cdot 1-3$ days after eclosion, adult offspring were transferred to $29^{\circ} \mathrm{C}$ to drive expression of UAS constructs ( 1 day at $29^{\circ} \mathrm{C}$ for all UAS constructs, except for optogenetic RhoGEF2 membrane recruitment (Figure 7D-F, which was induced for 2-3 days at $29^{\circ} \mathrm{C}$ ). To avoid unintended optogenetic system activation by light, we kept fly vials inside cardboard boxes or wrapped in aluminum foil and handled them in a dark room under a 593nm LED light source (SuperBrightLEDs) from this point forward. For coexpression of LARIAT with other UAS constructs, we used temperature-sensitive $G a / 80^{t s}$ to fully suppress premature Gal4-UAS driven transcription prior to temperature shift to $29^{\circ} \mathrm{C}$. To express LARIAT in clones, we generated Gal80 clones through FLP/FRT-mediated recombination [98]. These crosses were kept at $18^{\circ} \mathrm{C}$, protected from light and heat shocked at $37^{\circ} \mathrm{C}$ for 2 hours $3-5$ times. LARIAT-expressing cells were marked by the presence of 2 nlsRFP copies, while wild-type cells had either 1 or no nlsRFP copies. Alternatively, in Figure S2A, LARIAT-expressing cells were identified by the presence of GFP::aPKC clusters.

For in vivo optogenetic experiments, flies were exposed continuously to blue light for the indicated periods of time (in Table $\mathrm{S} 1$ and figure legends) by placing vials at approximately 8 $\mathrm{cm}$ from a $472 \mathrm{~nm}$ LED bulb (SuperBrightLEDs) at room temperature. Afterwards, we dissected their ovaries and fixed them. For each independent experiment, control flies from each genotype were kept in the dark and dissected in a dark room to avoid triggering CIBNCRY2 interaction. To control for potential blue light toxicity, we also exposed flies without optogenetic constructs to blue light using the same setup (data included in Figure 1).

For ex vivo optogenetic experiments, ovaries were dissected in a dark room in ex vivo culture medium (Schneider's medium (Sigma-Aldrich) supplemented with 10\% FBS (fetal bovine serum, heat inactivated; Thermo Fisher) and $200 \mu \mathrm{g} / \mathrm{mL}$ insulin (Sigma-Aldrich)). Afterwards, the dissected ovaries were transferred to new ex vivo culture medium and the ovarioles were partially separated by pipetting up and down gently. The separated ovarioles were exposed to blue light for 2 hours in 24-well-plates using the same setup as for whole flies and then they were fixed and stained to evaluate epithelial architecture. When indicated in the figures and figure legends, specific drugs (or DMSO or etanol for control samples) were added 20 minutes 
before exposure to blue light: Colchicine (Sigma-Aldrich; $30 \mu \mathrm{M}$; prepared in ethanol) to depolymerize microtubules and block cells in mitosis; Binucleine-2 (Sigma-Aldrich; $40 \mu \mathrm{M}$; prepared in DMSO) to inhibit Aurora B; and Latrunculin A to disrupt the actin cytoskeleton (Sigma Aldrich; $5 \mu \mathrm{g} / \mathrm{mL}$; prepared in DMSO). To confirm that Binucleine-2 blocked cytokinesis and Colchicine blocked cells in mitosis in the follicular epithelium, ovarioles were treated with these drugs for 30 minutes and then fixed (without exposing them to blue light). For live imaging, ovaries were dissected in a dark room and CIBN-CRY2 interaction was only triggered with the $488 \mathrm{~nm}$ laser used for GFP-tagged protein imaging.

\section{Fixation and staining of egg chambers}

778

To evaluate epithelial architecture, Drosophila ovaries were dissected in Schneider's medium (Sigma-Aldrich) supplemented with 10\% FBS (fetal bovine serum, heat inactivated; Thermo Fisher), washed once with PBT (PBS + 0.05\% Tween 20 (Sigma-Aldrich)) and fixed in 4\% paraformaldehyde solution (prepared in PBS with 0.2\% Tween 20 (Sigma-Aldrich)) for 20 min. For F-actin staining, Phalloidin-FITC (Sigma-Aldrich) or Phalloidin-TRITC (Sigma-Aldrich) was added to the fixative solution at $1 \mu \mathrm{g} / \mathrm{mL}$ and incubation time was increased to $30 \mathrm{~min}$. After washing three times for 10 min with PBT, samples were mounted with Vectashield with DAPI (Vector Laboratories). To evaluate mitotic progression (for Figure S3D), egg chambers were stained for Histone H3 phosphorylated Ser10 and actin. After fixation and washing, samples were blocked for 2 hours at room temperature with 10\% FBS (prepared in PBS $+0.2 \%$ Tween 20) and incubated overnight at $4^{\circ} \mathrm{C}$ with rabbit anti-phospho-Histone $\mathrm{H} 3(\mathrm{pH} 3) \operatorname{Ser} 10$ (1:2000; Upstate Biotechnology) diluted in PBT $+1 \%$ FBS. Afterwards, the samples were washed four times with PBT $+1 \%$ FBS for 30 minutes and incubated for two hours at room temperature with Alexa Fluor 568-conjugated goat anti-rabbit (Invitrogen; 1:300) diluted in PBT $+0.1 \%$ FBS. After washing two times with PBT for ten minutes, samples were incubated for 30 minutes with Phalloidin-TRITC diluted in PBT, washed three times for ten minutes and mounted with Vectashield with DAPI (Vector Laboratories).

\section{Optogenetic experiment and immunofluorescence in neuroblasts}

We used pnt-Gal4 to drive UAS-LARIAT expression in type II neuroblasts. Following a $12 \mathrm{~h}$ egg-laying period, control and LARIAT embryos were kept in the dark until wandering L3 larvae (wL3) stage. wL3 of both conditions were exposed to light for $1 \mathrm{~h}$. Brains were then dissected in PBS 1x, fixed in 4\% paraformaldehyde for $20 \mathrm{~min}$ at room temperature and washed three times with PBST (0.1\% Triton X-100 in $1 \times$ PBS). Brains were blocked with $1 \%$ normal goat serum in $0.1 \%$ PBST for at least 20 min at room temperature and incubated overnight at $4^{\circ} \mathrm{C}$ with rabbit anti-Miranda (1:2000, [99], gift from Juergen A. Knoblich) and mouse monoclonal anti-phospho-Histone $\mathrm{H} 3(\mathrm{pH} 3)$ Ser10 (1:1000, Cell Signalling, 9706), diluted in blocking solution. Afterwards, brains were washed three times, blocked for $20 \mathrm{~min}$ and incubated for $2 \mathrm{~h}$ at room temperature with secondary antibodies Alexa Fluor 647-conjugated goat anti-mouse and Alexa Fluor 568-conjugated goat anti-rabbit (Invitrogen), used at 1:1000. Finally, brains were mounted in Aqua Polymount (Polysciences, Inc).

of fixed Drosoph

811 Images of fixed Drosophila egg chambers were collected with a $1.1 \mathrm{NA} / 40 \mathrm{x}$ water or 1.30 812 NA/63x glycerine objectives on an inverted laser scanning confocal microscope Leica TCS 813 SP5 II (Leica Microsystems) or 1.30 NA/63x glycerol objective on an inverted laser scanning 
confocal microscope Leica SP8 (Leica Microsystems). To score epithelial defects and evaluate mitotic progression, images for egg chamber staging were collected with a $10 \mathrm{x}$ objective on a Zeiss Axio Imager Z1 microscope (Carl Zeiss, Germany) or a Zeiss Axio Imager Z1 Apotome microscope (Carl Zeiss, Germany). To evaluate epithelial architecture defects (epithelial gaps and/or multilayering), midsagittal cross-sections of egg chambers were inspected with a $20 x$ or $40 x$ Oil objective. To evaluate mitotic progression, images from the follicular epithelium at the surface of egg chambers were acquired with a 40x Oil objective on a Zeiss Axio Imager Z1 microscope (Carl Zeiss, Germany). Images from Drosophila larvae brains were acquired with a Zeiss LSM880 confocal microscope (Zeiss) using a LD LCI PlanApochromat 40x/1.2 Imm Corr DIC M27 water objective.

\section{Live imaging}

For live imaging of Drosophila egg chambers, individual ovarioles were dissected in ex vivo culture medium (Schneider's medium (Sigma-Aldrich) supplemented with 10\% FBS (fetal bovine serum, heat inactivated; Thermo Fisher) and $200 \mu \mathrm{g} / \mathrm{uL}$ insulin (Sigma-Aldrich)) and the enveloping muscle removed as previously described. Ovarioles were transferred to new culture medium and imaged on uncoated coverslips or glass bottom dishes (MatTek; No 1.5; P35G-1.5-7-C) with an Andor XD Revolution Spinning Disk Confocal system equipped with two solid state lasers - 488nm and $561 \mathrm{~nm}$-, an iXonEM+ DU-897 EMCCD camera and a Yokogawa CSU-22 unit built on an inverted Olympus IX81 microscope with a PLAPON 60x/NA 1.42 or a UPLSAPO 100x/NA 1.40 objective using iQ software (Andor). On average 2 egg chambers were imaged per fly. When indicated in the figures, to mark the cell membrane, ovarioles were stained with CellMask Orange Plasma membrane Stain (ThermoFisher; C10045; diluted 1:10 000 in culture medium) for 15 minutes and washed twice with ex vivo culture medium before imaging. Live imaging was performed at $25^{\circ} \mathrm{C}$. When indicated in the figures and figure legends, Colchicine (Sigma-Aldrich; $30 \mu \mathrm{M}$; prepared in etanol), Binucleine2 (Sigma-Aldrich; $40 \mu \mathrm{M}$; prepared in DMSO) or Latrunculin A (Sigma Aldrich; $5 \mu \mathrm{g} / \mathrm{mL}$; prepared in DMSO) were added at least 15 minutes before imaging. Midsagittal egg chamber cross-sections were used to image the follicular epithelium along the apical-basal axis and zstacks at the surface of the egg chamber to cross-section the follicular epithelium along the apical-basal axis.

For live imaging of larval brain neuroepithelia, brains from L3 larvae were dissected in Schneider's medium supplemented with glucose $(1 \mathrm{mg} / \mathrm{ml})$ and insulin $(0.2 \mathrm{mg} / \mathrm{ml})$ and transferred to a $10 \mu \mathrm{l}$ drop of the same medium supplemented with Fibrinogen $(0.2 \mathrm{mg} / \mathrm{ml})$ on a $25 \mathrm{~mm}$ glass-bottom dish. Brains were oriented on their side and the Fibrinogen was clotted using thrombin $(100 \mathrm{U} / \mathrm{ml}$, Sigma-Aldrich, T7513). After $3 \mathrm{~min}, 190 \mu \mathrm{l} \mathrm{Schneider's} \mathrm{medium}$ supplemented with glucose and insulin was pipetted on top of the clot. The neuroepithelia were imaged for 15 minutes on a Zeiss 710 Spinning Disk microscope using a 63x PlanApochromat 1.4 NA objective. $200 \mu \mathrm{l}$ Schneider's medium supplemented with glucose, insulin and 1NA-PP1 $(20 \mu \mathrm{M})$ was then added for a final concentration of $10 \mu \mathrm{M} 1 \mathrm{NA}-\mathrm{PP} 1$, after which imaging was resumed.

\section{Protein extracts and Western blot}

To confirm endogenous and GFP::aPKC levels in the different genotypes used for optogenetic aPKC inactivation (Figure S1A), we prepared protein extracts from Drosophila ovaries (at least 15 flies per genotype) dissected in a dark room. Dissected Drosophila ovaries were transferred to lysis buffer (150mM KCl, 75mM HEPES pH 7.5, $1.5 \mathrm{mM} \mathrm{EGTA,} 1.5 \mathrm{mM} \mathrm{MgCl} 2,15 \%$ glycerol, 
$8610.1 \%$ NP-40, $1 \times$ protease inhibitors cocktail (Roche) and 1x phosphatase inhibitors cocktail 3

862 (Sigma-Aldrich)), frozen in liquid nitrogen, thawed and then disrupted through sonication. We

863 clarified lysates through two consecutive centrifugations at $14000 \mathrm{rpm}$ for $10 \mathrm{~min}$ at $4^{\circ} \mathrm{C}$.

864 Protein concentration was determined with NanoDrop 1000 Spectrophotometer (Thermo

865 Fisher). Samples were then resolved through SDS-PAGE and transferred to a nitrocellulose

866 membrane using the iBlot Dry Blotting System (Invitrogen) for Western blotting. Protein

867 transfer was confirmed by Ponceau staining (0.25\% Ponceau S in $40 \%$ methanol and $15 \%$

868 acetic acid). The membranes were blocked for two hours at room temperature with $5 \%$ dry

869 milk prepared in PBT and incubated overnight at $4^{\circ} \mathrm{C}$ with the primary antibodies (rabbit anti-

870 aPKC 1:2000 (c-20, Santa Cruz Biotechnology) and mouse anti- $\alpha$-Tubulin 1:10 000 (DM1A,

871 Santa Cruz Biotechnology)) diluted in PBT $+1 \%$ dry milk. After washing three times for 10 min

872 with PBT, membranes were incubated with the secondary antibodies anti-mouse and anti-

873 rabbit conjugated with horseradish peroxidase diluted in PBT $+1 \%$ dry milk for one hour at

874 room temperature. After washing again three times for 10 min with PBT, blots were developed

875 with ECL Chemiluminescent Detection System (Amersham) according to the manufacturer's

876 instructions and revealed with a ChemiDoc XRS+ (BioRad).

877

\section{aPKC ${ }^{\text {as } 4}$ allele inactivation}

879 For epithelial defect analysis in the follicular epithelium, Drosophila ovaries from aPKC ${ }^{\text {as } 4}$ flies 880 (prepared as previously described in the optogenetic experiments section) were cultured ex 881 vivo for 2 hours in the presence of the ATP analog 1NA-PP1 (Calbiochem; prepared in DMSO; 882 at the concentrations indicated in Figure 2 and the respective figure legend) before fixation. 883 DMSO was added to control samples. For live imaging of egg chambers and larval 884 neuroepithelium, 1NA-PP1 (at the concentration indicated in figure legends) or DMSO was 885 added to the culture medium at the indicated timing.

\section{QUANTIFICATION AND STATISTICAL ANALYSIS}

Image processing and quantifications were done with FIJI [100]. Statistical analysis and graphs were done in GraphPad Prism 8 (GraphPad Software Inc., La Jolla, CA, USA), except when otherwise indicated.

\section{Epithelial defects analysis}

To evaluate epithelial architecture, we scored the amount of egg chambers at specific developmental stages with one or more epithelial gaps, one or more multilayering events or both in midsagittal cross-sections. As egg chambers develop, they grow in size. Thus, we determined the developmental stage of egg chambers by measuring their area in midsagittal cross-sections, as a proxy for size. To define the area intervals corresponding to each developmental stage, we staged control egg chambers from GFP::aPKC flies according to phenotypic characteristics, as in Jia et al., [101], and correlated their stage with their size. We scored epithelial defects (epithelial gaps and/or multilayering) and their position (anterior, posterior, dorsal-ventral) by inspecting midsagittal cross-sections of egg chambers: for LARIAT and $\mathrm{aPKC}^{\text {as4 }}$ experiments, egg chambers were stained with DAPI (DNA) and Phalloidin (F-actin); for optogenetic RhoGEF2 membrane recruitment, egg chambers were stained with DAPI (DNA) and PatJ-CIBN-pmGFP and RhoGEF2-CRY2-mCherry fluorescence was used. For the initial analysis of aPKC inactivation with LARIAT (Figure 1C), results from 
905

3 independent experiments ( $\geq 8$ flies per condition per experiment) were summed up in a single contingency table and the graph shows the relative amount of egg chambers (stages 3 to 8 ) with each type of defect found. For statistical analysis, epithelial gaps and/or multilayering were grouped in a single defect category and Fisher's exact test with Bonferroni correction for multiple comparison was used. For other experiments, graphs show mean percentage of egg chambers with the indicated type of defect \pm standard deviation (SD). The percentages of defective egg chambers obtained for each independent experiment for each condition $(\geq 8$ flies per condition per independent experiment) are represented as individual data points in the graphs. The total amount of egg chambers scored in each analysis is indicated in the respective graph as $n$. To ensure consistent LARIAT expression levels, only proliferative stages 4 to 6 were included in analyses (except in Figures 1 and S3B). To test for statistical significance, we built contingency tables comparing the sum of egg chambers from all replicates with one or more epithelial gap vs no gap and used Fisher's exact test, with Bonferroni correction for multiple comparisons when necessary. To compare the frequency of epithelial gap and multilayering formation at the anterior, posterior and dorsal-ventral regions, we analyzed how many of the epithelial gaps and multilayering events detected upon aPKC clustering in proliferative egg chambers were present at these different regions irrespective of how long the samples had been exposed to blue light (data in Fig. 2C).

\section{Lgl asymmetry in the follicular epithelium}

The ratio of apical over lateral $\mathrm{Lgl}$ fluorescence intensity was used to analyse the asymmetric distribution of $\mathrm{Lgl}$ along the apical-basal axis in the follicular epithelium after aPKC clustering. For each egg chamber, the lateral and apical cortex of all epithelial cells was manually segmented, average fluorescence intensity was extracted and corrected for average Lgl intensity in the cytoplasm. Parts of the epithelium presenting with multilayering were excluded from the analysis (at least 10 cells per egg chamber were included in the analysis). Each point in Figure $1 \mathrm{E}$ represents the apical/lateral Lgl ratio for an individual egg chamber.

\section{Miranda asymmetry in neuroblasts}

The distribution of Miranda along the membrane of dividing NBs ( $\mathrm{pH} 3$ positive) was analysed. To extract Miranda intensity profile, that is, the intensity of Miranda $\left(I_{M}\right)$ along the length of the cell membrane (L), we proceeded as in (Rodriguez et al., 2017) with minor changes: we used a 30-pixel wide stripe to delineate the membrane; profile extraction was initiated in the basal membrane section, so that $L<50 \%$ correspond to basal Miranda intensity values. In the end, Miranda intensity $\left(\mathrm{I}_{\mathrm{M}}\right)$ was plotted as a function of percentage of membrane length $(\mathrm{L})$. To obtain the asymmetry index (ASI) for Miranda, Basal (B) and Apical (A) intensities were calculated as the area under the Miranda intensity plot $\left(I_{M}\right)$ for the basal $(L \leq 50 \%)$ and apical $(L>50 \%)$ sections of the membrane. Absolute ASI values were calculated as in (Hannaford et al., 2019) with the following formula:

\footnotetext{
ASI values were then normalized relative to control mean so that, an ASI of 1 represents normal asymmetry and lower values ( $\sim)$ indicate loss of asymmetry. As pnt-GAL4 only drives
} 
LARIAT expression in type II neuroblasts, only this subtype was considered in all calculations. Statistical analysis was performed using Prism 6 (GraphPad Software Inc., La Jolla, CA,

948 USA), mean \pm SD are depicted and individual ASI values represented (normalized to control 949 mean). Statistical significance of the difference of means was calculated using unpaired $t$-test 950 and considered significant when $p<0.05$.

\section{Apical myosin II in the follicular epithelium}

952 To quantify apical accumulation of myosin II after aPKC inactivation, we measured 953 Sqh::mKate2x3 fluorescence intensity at the apical domain of follicular epithelial cells in single 954 plane midsagittal cross-sections of egg chambers acquired during live imaging. For each egg 955 chamber, 4 regions of interest (ROIs), each one encompassing the apical domain of at least 9564 cells in a different region (dorsal, ventral, anterior or posterior), were manually defined and 957 tracked through time. Mean apical fluorescence intensity (AFI) for each timepoint was 958 extracted from raw movie datasets, corrected for mean cytoplasm fluorescence intensity (CFI; 959 average value measured for each timepoint in 3 follicular epithelial cells) and normalized to 960 the corrected fluorescence intensity before aPKC inactivation $\left(\mathrm{AFI}_{\text {initial }}-\mathrm{CFI}_{\text {initial }}\right)$ as follows:

For aPKC LARIAT experiments, $\mathrm{AFI}_{\text {initial }}-\mathrm{CF} \mathrm{I}_{\text {initial }}$ corresponds to the $\mathrm{AFI}-\mathrm{CFI}$ value at min 0 obtained by averaging AFI-CFI for the 5 frames before aPKC inactivation with 1NA-PP1 at min 0 . Whenever, an epithelial gap started forming at a particular region, quantification at that same region was stopped.

\section{Egg chamber circularity}

968 To assess egg chamber deformation after aPKC inactivation, we measured egg chamber circularity in single plane midsagittal cross-sections of Sqh::mKate $2 \times 3$ egg chambers acquired during live imaging. The apical surface of the follicular epithelium was manually segmented and circularity $\left(4 \pi\left(\right.\right.$ area/perimeter $\left.\left.{ }^{2}\right)\right)$ was measured. Egg chamber circularity was only quantified while no epithelial defect appeared.

\section{Apical surface area and myosin II in the neuroepithelium}

To measure apical surface contraction in the neuroepithelium upon aPKC ${ }^{\text {as } 4}$ inactivation with 1NA-PP1, the ROI edges were manually tracked as red dots using aPKC ${ }^{\text {as }}$-mScarlet signal to detect apical cell edges. These dots were then connected using a steerable filter for line detection. The resulting shape was then filled and dilated (blue mask in Figure $3 \mathrm{G}$ ) to approximate the area to measure. Apical area was normalized to its initial value at min 0 . To measure myosin II intensity at the apicomedial region and apical junctions after aPKC ${ }^{\text {as } 4}$ inactivation with 1NA-PP1, apical junctions were segmented using aPKC ${ }^{\text {as }}$-mScarlet signal. aPKC signal to noise ratio was increased by a steerable filter detecting lines and a junctional mask (as the one shown in Figure $3 \mathrm{~J}$ ) was generated by thresholding. The mask for the 
apicomedial region was generated by inverting and contracting the junctional mask. Myosin II intensity was obtained by extracting average Zip::YFP intensity with these masks and normalizing to junctional intensity at min -2 before aPKC inactivation.

\section{Apical area in interphase and mitotic cells in the follicular epithelium}

To evaluate apical constriction in interphase cells and pulling forces on mitotic cells, we measured epithelial cell area in cross-sections at the junctional level of the follicular epithelium acquired during live imaging. For each egg chamber, we quantified the mean apical cell area (average of at least 3 interphase cells manually segmented using E-cad::mKate2 per egg chamber). Surface area was normalized to the initial mean value, obtained by averaging the corresponding cell area for the 3 frames before aPKC clustering (from min -2 to 0 ). A similar procedure was used to segment cells that entered mitosis up to 5 minutes after aPKC clustering was initiated. Anaphase onset was defined as the first frame of cell elongation (determined through E-cad::mKate2 signal at the lateral cortex). Mitotic entry was defined as the first frame of visible mitotic rounding in a lateral cortex cross-section.

To evaluate apical constriction in clones of LARIAT-expressing cells, we measured the epithelial cell area at the apical surface of the follicular epithelium acquired during live imaging. For each clone, we quantified the mean apical cell area (average of at least 6 cells per clone manually segmented in 4D stacks using GFP::aPKC and a plasma membrane marker). A similar procedure was used to measure the apical area of individual wild-type cells adjacent to LARIAT-expressing cells (Figure 7C).

\section{Epithelial gap analysis live}

To evaluate whether and where gaps formed in the follicular epithelium, 4D stacks of surface cross-sections from egg chambers stained with membrane marker were analyzed. Gaps were inspected to verify if they span the whole length of the apical-basal axis and were only included in the analysis when all neighbour cells were in sight, so as to be able to determine whether any of them were in mitosis. The number of independent gaps detected in the 13 control, 8 Binucleine-2-treated and 6 Colchicine-treated egg chambers is indicated as $n$ in Figures 5B, $5 \mathrm{G}$ and $5 \mathrm{I}$, respectively.

\section{Mitotic progression}

To confirm the effect of Binucleine-2 and Colchicine, we analyzed mitotic progression in control and drug treated egg chambers. Mitotic cells were identified through positive staining with $\mathrm{pH} 3$ (number of cells counted indicated as $\mathrm{n}$ in Figure S3D and E). DAPI staining was used to verify whether sister chromatids had separated and group cells into early mitosis (prophase, prometaphse, metaphase) and late mitosis (anaphase, telophase) or cytokinesis. DNA. Actin staining was used to verify whether cells had elongated, confirming anaphase onset, and whether they had assembled a cytokinetic ring.

\section{Image preparation}

Representative images were processed and prepared using FIJI. Representative midsagittal images from egg chambers are from a single optical section or 2-5 plane maximum projection. Surface images from egg chambers are maximum projections of all optical sections covering the epithelial domain of interest. When necessary, movies were registered with the FIJI plugin 
StackReg (EPFL; Biomedical Imaging Group), to correct for whole egg chamber movement during live imaging; and a Gaussian Blur or Gaussian Blur 3D filter was applied to remove random noise.

\section{References}

1. Munjal, A., and Lecuit, T. (2014). Actomyosin networks and tissue morphogenesis. Development 141, 1789-1793.

2. Takeichi, M. (2014). Dynamic contacts: rearranging adherens junctions to drive epithelial remodelling. Nat Rev Mol Cell Biol 15, 397-410.

3. Halaoui, R., Rejon, C., Chatterjee, S.J., Szymborski, J., Meterissian, S., Muller, W.J., Omeroglu, A., and McCaffrey, L. (2017). Progressive polarity loss and luminal collapse disrupt tissue organization in carcinoma. Genes Dev. 31, 1573-1587.

4. Bilder, D., Li, M., and Perrimon, N. (2000). Cooperative regulation of cell polarity and growth by Drosophila tumor suppressors. Science 289, 113-116.

5. Horne-Badovinac, S., Lin, D., Waldron, S., Schwarz, M., Mbamalu, G., Pawson, T., Jan, Y., Stainier, D.Y., and Abdelilah-Seyfried, S. (2001). Positional cloning of heart and soul reveals multiple roles for PKC lambda in zebrafish organogenesis. Curr Biol 11, 1492-1502.

6. Wodarz, A., Ramrath, A., Grimm, A., and Knust, E. (2000). Drosophila atypical protein kinase $\mathrm{C}$ associates with Bazooka and controls polarity of epithelia and neuroblasts. $\mathrm{J}$ Cell Biol 150, 1361-1374.

7. Imai, F., Hirai, S., Akimoto, K., Koyama, H., Miyata, T., Ogawa, M., Noguchi, S., Sasaoka, T., Noda, T., and Ohno, S. (2006). Inactivation of aPKClambda results in the loss of adherens junctions in neuroepithelial cells without affecting neurogenesis in mouse neocortex. Development 133, 1735-1744.

8. Tanentzapf, G., Smith, C., McGlade, J., and Tepass, U. (2000). Apical, lateral, and basal polarization cues contribute to the development of the follicular epithelium during Drosophila oogenesis. J Cell Biol 151, 891-904.

9. Sallee, M.D., Pickett, M.A., and Feldman, J.L. (2021). Apical PAR complex proteins protect against programmed epithelial assaults to create a continuous and functional intestinal lumen. Elife 10.

10. Whiteman, E.L., Fan, S., Harder, J.L., Walton, K.D., Liu, C.J., Soofi, A., Fogg, V.C., Hershenson, M.B., Dressler, G.R., Deutsch, G.H., et al. (2014). Crumbs3 is essential for proper epithelial development and viability. Mol Cell Biol 34, 43-56.

11. Guilgur, L.G., Prudencio, P., Ferreira, T., Pimenta-Marques, A.R., and Martinho, R.G. (2012). Drosophila aPKC is required for mitotic spindle orientation during symmetric division of epithelial cells. Development 139, 503-513.

12. Seidl, S., Braun, U., Roos, N., Li, S., Ludtke, T.H., Kispert, A., and Leitges, M. (2013). Phenotypical analysis of atypical PKCs in vivo function display a compensatory system at mouse embryonic day 7.5. PLoS One 8, e62756.

13. Rodriguez-Boulan, E., and Macara, I.G. (2014). Organization and execution of the epithelial polarity programme. Nat Rev Mol Cell Biol 15, 225-242.

14. Hong, Y. (2018). aPKC: the Kinase that Phosphorylates Cell Polarity. F1000Res 7.

15. Morais-de-Sa, E., Mirouse, V., and St Johnston, D. (2010). aPKC phosphorylation of Bazooka defines the apical/lateral border in Drosophila epithelial cells. Cell 141, 509-523.

16. Suzuki, A., Hirata, M., Kamimura, K., Maniwa, R., Yamanaka, T., Mizuno, K., Kishikawa, M., Hirose, H., Amano, Y., Izumi, N., et al. (2004). aPKC acts upstream of PAR- 
1070

1071

1072

1073

1074

1075

1076

1077

1078

1079

1080

1081

1082

1083

1084

1085

1086

1087

1088

1089

1090

1091

1092

1093

1094

1095

1096

1097

1098

1099

1100

1101

1102

1103

1104

1105

1106

1107

1108

1109

1110

1111

1112

1113

1114

1115

1116

1117

$1 \mathrm{~b}$ in both the establishment and maintenance of mammalian epithelial polarity. Curr Biol 14 , 1425-1435.

17. Krahn, M.P., Buckers, J., Kastrup, L., and Wodarz, A. (2010). Formation of a BazookaStardust complex is essential for plasma membrane polarity in epithelia. J Cell Biol 190, 751760 .

18. Goransson, O., Deak, M., Wullschleger, S., Morrice, N.A., Prescott, A.R., and Alessi, D.R. (2006). Regulation of the polarity kinases PAR-1/MARK by 14-3-3 interaction and phosphorylation. J Cell Sci 119, 4059-4070.

19. Gamblin, C.L., Parent-Prevost, F., Jacquet, K., Biehler, C., Jette, A., and Laprise, P. (2018). Oligomerization of the FERM-FA protein Yurt controls epithelial cell polarity. J Cell Biol 217, 3853-3862.

20. Soriano, E.V., Ivanova, M.E., Fletcher, G., Riou, P., Knowles, P.P., Barnouin, K., Purkiss, A., Kostelecky, B., Saiu, P., Linch, M., et al. (2016). aPKC Inhibition by Par3 CR3 Flanking Regions Controls Substrate Access and Underpins Apical-Junctional Polarization. Dev Cell 38, 384-398.

21. Bailey, M.J., and Prehoda, K.E. (2015). Establishment of Par-Polarized Cortical Domains via Phosphoregulated Membrane Motifs. Developmental cell 35, 199-210.

22. Dong, W., Zhang, X., Liu, W., Chen, Y.J., Huang, J., Austin, E., Celotto, A.M., Jiang, W.Z., Palladino, M.J., Jiang, Y., et al. (2015). A conserved polybasic domain mediates plasma membrane targeting of $\mathrm{Lgl}$ and its regulation by hypoxia. The Journal of cell biology $211,273-$ 286.

23. Iden, S., Misselwitz, S., Peddibhotla, S.S., Tuncay, H., Rehder, D., Gerke, V., Robenek, H., Suzuki, A., and Ebnet, K. (2012). aPKC phosphorylates JAM-A at Ser285 to promote cell contact maturation and tight junction formation. J Cell Biol 196, 623-639.

24. Niessen, M.T., Scott, J., Zielinski, J.G., Vorhagen, S., Sotiropoulou, P.A., Blanpain, C., Leitges, M., and Niessen, C.M. (2013). aPKClambda controls epidermal homeostasis and stem cell fate through regulation of division orientation. J Cell Biol 202, 887-900.

25. Harris, T.J., and Peifer, M. (2007). aPKC controls microtubule organization to balance adherens junction symmetry and planar polarity during development. Dev Cell 12, 727-738.

26. Ishiuchi, T., and Takeichi, M. (2011). Willin and Par3 cooperatively regulate epithelial apical constriction through aPKC-mediated ROCK phosphorylation. Nat Cell Biol 13, 860-866.

27. Dias Gomes, M., Letzian, S., Saynisch, M., and Iden, S. (2019). Polarity signaling ensures epidermal homeostasis by coupling cellular mechanics and genomic integrity. Nat Commun 10, 3362.

28. Franco, M., and Carmena, A. (2019). Eph signaling controls mitotic spindle orientation and cell proliferation in neuroepithelial cells. J Cell Biol 218, 1200-1217.

29. Hao, Y., Du, Q., Chen, X., Zheng, Z., Balsbaugh, J.L., Maitra, S., Shabanowitz, J., Hunt, D.F., and Macara, I.G. (2010). Par3 controls epithelial spindle orientation by aPKCmediated phosphorylation of apical Pins. Curr Biol 20, 1809-1818.

30. Jung, H.Y., Fattet, L., Tsai, J.H., Kajimoto, T., Chang, Q., Newton, A.C., and Yang, J. (2019). Apical-basal polarity inhibits epithelial-mesenchymal transition and tumour metastasis by PAR-complex-mediated SNAI1 degradation. Nat Cell Biol 21, 359-371.

31. Korotkevich, E., Niwayama, R., Courtois, A., Friese, S., Berger, N., Buchholz, F., and Hiiragi, T. (2017). The Apical Domain Is Required and Sufficient for the First Lineage Segregation in the Mouse Embryo. Dev Cell 40, 235-247 e237.

32. Sabherwal, N., Thuret, R., Lea, R., Stanley, P., and Papalopulu, N. (2014). aPKC phosphorylates p27Xic1, providing a mechanistic link between apicobasal polarity and cellcycle control. Dev Cell 31, 559-571. 
33. Matsuzawa, K., Ohga, H., Shigetomi, K., Shiiya, T., Hirashima, M., and Ikenouchi, J. (2021). MAGIs regulate aPKC to enable balanced distribution of intercellular tension for epithelial sheet homeostasis. Commun Biol 4, 337.

34. Zihni, C., Vlassaks, E., Terry, S., Carlton, J., Leung, T.K.C., Olson, M., Pichaud, F., Balda, M.S., and Matter, K. (2017). An apical MRCK-driven morphogenetic pathway controls epithelial polarity. Nat Cell Biol 19, 1049-1060.

35. David, D.J., Tishkina, A., and Harris, T.J. (2010). The PAR complex regulates pulsed actomyosin contractions during amnioserosa apical constriction in Drosophila. Development 137, 1645-1655.

36. Durney, C.H., Harris, T.J.C., and Feng, J.J. (2018). Dynamics of PAR Proteins Explain the Oscillation and Ratcheting Mechanisms in Dorsal Closure. Biophys J 115, 2230-2241.

37. Rosa, A., Vlassaks, E., Pichaud, F., and Baum, B. (2015). Ect2/Pbl acts via Rho and polarity proteins to direct the assembly of an isotropic actomyosin cortex upon mitotic entry. Developmental cell 32, 604-616.

38. Morais-de-Sa, E., and Sunkel, C. (2013). Adherens junctions determine the apical position of the midbody during follicular epithelial cell division. EMBO Rep 14, 696-703.

39. Ragkousi, K., Marr, K., McKinney, S., Ellington, L., and Gibson, M.C. (2017). CellCycle-Coupled Oscillations in Apical Polarity and Intercellular Contact Maintain Order in Embryonic Epithelia. Curr Biol 27, 1381-1386.

40. Zenker, J., White, M.D., Gasnier, M., Alvarez, Y.D., Lim, H.Y.G., Bissiere, S., Biro, M., and Plachta, N. (2018). Expanding Actin Rings Zipper the Mouse Embryo for Blastocyst Formation. Cell 173, 776-791 e717.

41. Osswald, M., and Morais-de-Sa, E. (2019). Dealing with apical-basal polarity and intercellular junctions: a multidimensional challenge for epithelial cell division. Curr Opin Cell Biol 60, 75-83.

42. Lee, S., Park, H., Kyung, T., Kim, N.Y., Kim, S., Kim, J., and Heo, W.D. (2014). Reversible protein inactivation by optogenetic trapping in cells. Nat Methods 11, 633-636.

43. Chen, J., Sayadian, A.C., Lowe, N., Lovegrove, H.E., and St Johnston, D. (2018). An alternative mode of epithelial polarity in the Drosophila midgut. PLoS Biol 16, e3000041.

44. Qin, X., Park, B.O., Liu, J., Chen, B., Choesmel-Cadamuro, V., Belguise, K., Heo, W.D., and Wang, X. (2017). Cell-matrix adhesion and cell-cell adhesion differentially control basal myosin oscillation and Drosophila egg chamber elongation. Nat Commun 8, 14708.

45. Kim, S., Gailite, I., Moussian, B., Luschnig, S., Goette, M., Fricke, K., HonemannCapito, M., Grubmuller, H., and Wodarz, A. (2009). Kinase-activity-independent functions of atypical protein kinase $C$ in Drosophila. J Cell Sci 122, 3759-3771.

46. Franz, A., and Riechmann, V. (2010). Stepwise polarisation of the Drosophila follicular epithelium. Dev Biol 338, 136-147.

47. Rolls, M.M., Albertson, R., Shih, H.P., Lee, C.Y., and Doe, C.Q. (2003). Drosophila aPKC regulates cell polarity and cell proliferation in neuroblasts and epithelia. J Cell Biol 163, 1089-1098.

48. Hannaford, M., Loyer, N., Tonelli, F., Zoltner, M., and Januschke, J. (2019). A chemical-genetics approach to study the role of atypical Protein Kinase $C$ in Drosophila. Development 146.

49. Vasquez, C.G., Heissler, S.M., Billington, N., Sellers, J.R., and Martin, A.C. (2016). Drosophila non-muscle myosin II motor activity determines the rate of tissue folding. Elife 5 .

50. Munjal, A., Philippe, J.M., Munro, E., and Lecuit, T. (2015). A self-organized biomechanical network drives shape changes during tissue morphogenesis. Nature 524, 351355. 
1166

1167

1168

1169

1170

1171

1172

1173

1174

1175

1176

1177

1178

1179

1180

1181

1182

1183

1184

1185

1186

1187

1188

1189

1190

1191

1192

1193

1194

1195

1196

1197

1198

1199

1200

1201

1202

1203

1204

1205

1206

1207

1208

1209

1210

1211

1212

1213

51. Kasza, K.E., Farrell, D.L., and Zallen, J.A. (2014). Spatiotemporal control of epithelial remodeling by regulated myosin phosphorylation. Proc Natl Acad Sci U S A 111, 1173211737.

52. Smurnyy, Y., Toms, A.V., Hickson, G.R., Eck, M.J., and Eggert, U.S. (2010). Binucleine 2, an isoform-specific inhibitor of Drosophila Aurora B kinase, provides insights into the mechanism of cytokinesis. ACS Chem Biol 5, 1015-1020.

53. Mishima, M., Kaitna, S., and Glotzer, M. (2002). Central spindle assembly and cytokinesis require a kinesin-like protein/RhoGAP complex with microtubule bundling activity. Dev Cell 2, 41-54.

54. Alegot, H., Pouchin, P., Bardot, O., and Mirouse, V. (2018). Jak-Stat pathway induces Drosophila follicle elongation by a gradient of apical contractility. Elife 7.

55. Krueger, D., Tardivo, P., Nguyen, C., and De Renzis, S. (2018). Downregulation of basal myosin-II is required for cell shape changes and tissue invagination. EMBO J 37.

56. Maitre, J.L., Turlier, H., Illukkumbura, R., Eismann, B., Niwayama, R., Nedelec, F., and Hiiragi, T. (2016). Asymmetric division of contractile domains couples cell positioning and fate specification. Nature 536, 344-348.

57. David, D.J., Wang, Q., Feng, J.J., and Harris, T.J. (2013). Bazooka inhibits aPKC to limit antagonism of actomyosin networks during amnioserosa apical constriction. Development 140, 4719-4729.

58. Sidor, C., Stevens, T.J., Jin, L., Boulanger, J., and Roper, K. (2020). Rho-Kinase Planar Polarization at Tissue Boundaries Depends on Phospho-regulation of Membrane Residence Time. Dev Cell 52, 364-378 e367.

59. Flores-Benitez, D., and Knust, E. (2015). Crumbs is an essential regulator of cytoskeletal dynamics and cell-cell adhesion during dorsal closure in Drosophila. Elife 4.

60. Letizia, A., Sotillos, S., Campuzano, S., and Llimargas, M. (2011). Regulated Crb accumulation controls apical constriction and invagination in Drosophila tracheal cells. J Cell Sci 124, 240-251.

61. Nakajima, H., and Tanoue, T. (2011). Lulu2 regulates the circumferential actomyosin tensile system in epithelial cells through p114RhoGEF. J Cell Biol 195, 245-261.

62. Silver, J.T., Wirtz-Peitz, F., Simoes, S., Pellikka, M., Yan, D., Binari, R., Nishimura, T., Li, Y., Harris, T.J.C., Perrimon, N., et al. (2019). Apical polarity proteins recruit the RhoGEF Cysts to promote junctional myosin assembly. J Cell Biol 218, 3397-3414.

63. Biehler, C., Rothenberg, K.E., Jette, A., Gaude, H.M., Fernandez-Gonzalez, R., and Laprise, P. (2021). Pak1 and PP2A antagonize aPKC function to support cortical tension induced by the Crumbs-Yurt complex. Elife 10.

64. Finegan, T.M., Na, D., Cammarota, C., Skeeters, A.V., Nadasi, T.J., Dawney, N.S., Fletcher, A.G., Oakes, P.W., and Bergstralh, D.T. (2019). Tissue tension and not interphase cell shape determines cell division orientation in the Drosophila follicular epithelium. EMBO J 38.

65. Crest, J., Diz-Munoz, A., Chen, D.Y., Fletcher, D.A., and Bilder, D. (2017). Organ sculpting by patterned extracellular matrix stiffness. Elife 6 .

66. Borreguero-Munoz, N., Fletcher, G.C., Aguilar-Aragon, M., Elbediwy, A., VincentMistiaen, Z.I., and Thompson, B.J. (2019). The Hippo pathway integrates PI3K-Akt signals with mechanical and polarity cues to control tissue growth. PLoS Biol 17, e3000509.

67. Balaji, R., Weichselberger, V., and Classen, A.K. (2019). Response of Drosophila epithelial cell and tissue shape to external forces in vivo. Development 146.

68. Manning, L.A., Perez-Vale, K.Z., Schaefer, K.N., Sewell, M.T., and Peifer, M. (2019). The Drosophila Afadin and ZO-1 homologues Canoe and Polychaetoid act in parallel to 
maintain epithelial integrity when challenged by adherens junction remodeling. Mol Biol Cell 30, 1938-1960.

69. Monster, J.L., Donker, L., Vliem, M.J., Win, Z., Matthews, H.K., Cheah, J.S., Yamada, S., de Rooij, J., Baum, B., and Gloerich, M. (2021). An asymmetric junctional mechanoresponse coordinates mitotic rounding with epithelial integrity. J Cell Biol 220.

70. Ko, C.S., Kalakuntla, P., and Martin, A.C. (2020). Apical Constriction Reversal upon Mitotic Entry Underlies Different Morphogenetic Outcomes of Cell Division. Mol Biol Cell 31, 1663-1674.

71. Godard, B.G., Dumollard, R., Munro, E., Chenevert, J., Hebras, C., McDougall, A., and Heisenberg, C.P. (2020). Apical Relaxation during Mitotic Rounding Promotes TensionOriented Cell Division. Dev Cell.

72. Gupta, V.K., Nam, S., Yim, D., Camuglia, J., Martin, J.L., Sanders, E.N., O'Brien, L.E., Martin, A.C., Kim, T., and Chaudhuri, O. (2021). The nature of cell division forces in epithelial monolayers. J Cell Biol 220.

73. Aguilar-Aragon, M., Bonello, T.T., Bell, G.P., Fletcher, G.C., and Thompson, B.J. (2020). Adherens junction remodelling during mitotic rounding of pseudostratified epithelial cells. EMBO Rep 21, e49700.

74. Petridou, N.I., Grigolon, S., Salbreux, G., Hannezo, E., and Heisenberg, C.P. (2019). Fluidization-mediated tissue spreading by mitotic cell rounding and non-canonical Wnt signalling. Nat Cell Biol 21, 169-178.

75. Guillot, C., and Lecuit, T. (2013). Adhesion disengagement uncouples intrinsic and extrinsic forces to drive cytokinesis in epithelial tissues. Dev Cell 24, 227-241.

76. Higashi, T., Arnold, T.R., Stephenson, R.E., Dinshaw, K.M., and Miller, A.L. (2016). Maintenance of the Epithelial Barrier and Remodeling of Cell-Cell Junctions during Cytokinesis. Current biology : CB 26, 1829-1842.

77. Herszterg, S., Leibfried, A., Bosveld, F., Martin, C., and Bellaiche, Y. (2013). Interplay between the dividing cell and its neighbors regulates adherens junction formation during cytokinesis in epithelial tissue. Dev Cell 24, 256-270.

78. Founounou, N., Loyer, N., and Le Borgne, R. (2013). Septins regulate the contractility of the actomyosin ring to enable adherens junction remodeling during cytokinesis of epithelial cells. Dev. Cell 24, 242-255.

79. Gilmour, D., Rembold, M., and Leptin, M. (2017). From morphogen to morphogenesis and back. Nature 541, 311-320.

80. Campinho, P., Behrndt, M., Ranft, J., Risler, T., Minc, N., and Heisenberg, C.P. (2013). Tension-oriented cell divisions limit anisotropic tissue tension in epithelial spreading during zebrafish epiboly. Nat Cell Biol 15, 1405-1414.

81. Petridou, N.I., Corominas-Murtra, B., Heisenberg, C.P., and Hannezo, E. (2021). Rigidity percolation uncovers a structural basis for embryonic tissue phase transitions. Cell 184, 1914-1928 e1919.

82. Saadaoui, M., Rocancourt, D., Roussel, J., Corson, F., and Gros, J. (2020). A tensile ring drives tissue flows to shape the gastrulating amniote embryo. Science 367, 453-458.

83. Hoijman, E., Rubbini, D., Colombelli, J., and Alsina, B. (2015). Mitotic cell rounding and epithelial thinning regulate lumen growth and shape. Nat Commun 6, 7355.

84. Kondo, T., and Hayashi, S. (2013). Mitotic cell rounding accelerates epithelial invagination. Nature 494, 125-129.

85. Guirao, B., Rigaud, S.U., Bosveld, F., Bailles, A., Lopez-Gay, J., Ishihara, S., Sugimura, K., Graner, F., and Bellaiche, Y. (2015). Unified quantitative characterization of epithelial tissue development. Elife 4. 
86. Firmino, J., Rocancourt, D., Saadaoui, M., Moreau, C., and Gros, J. (2016). Cell Division Drives Epithelial Cell Rearrangements during Gastrulation in Chick. Dev Cell 36, 249261.

87. Mata, J., Curado, S., Ephrussi, A., and Rorth, P. (2000). Tribbles coordinates mitosis and morphogenesis in Drosophila by regulating string/CDC25 proteolysis. Cell 101, 511-522. 88. Seher, T.C., and Leptin, M. (2000). Tribbles, a cell-cycle brake that coordinates proliferation and morphogenesis during Drosophila gastrulation. Curr Biol 10, 623-629.

89. Grosshans, J., and Wieschaus, E. (2000). A genetic link between morphogenesis and cell division during formation of the ventral furrow in Drosophila. Cell 101, 523-531.

90. Wirtz-Peitz, F., Nishimura, T., and Knoblich, J.A. (2008). Linking cell cycle to asymmetric division: Aurora-A phosphorylates the Par complex to regulate Numb localization. Cell 135, 161-173.

91. Pandey, R., Heidmann, S., and Lehner, C.F. (2005). Epithelial re-organization and dynamics of progression through mitosis in Drosophila separase complex mutants. J Cell Sci $118,733-742$.

92. Pinheiro, D., Hannezo, E., Herszterg, S., Bosveld, F., Gaugue, I., Balakireva, M., Wang, Z., Cristo, I., Rigaud, S.U., Markova, O., et al. (2017). Transmission of cytokinesis forces via E-cadherin dilution and actomyosin flows. Nature 545, 103-107.

93. Huang, J., Zhou, W., Dong, W., Watson, A.M., and Hong, Y. (2009). From the Cover: Directed, efficient, and versatile modifications of the Drosophila genome by genomic engineering. Proc Natl Acad Sci U S A 106, 8284-8289.

94. Lowe, N., Rees, J.S., Roote, J., Ryder, E., Armean, I.M., Johnson, G., Drummond, E., Spriggs, H., Drummond, J., Magbanua, J.P., et al. (2014). Analysis of the expression patterns, subcellular localisations and interaction partners of Drosophila proteins using a pigP protein trap library. Development 141, 3994-4005.

95. Izquierdo, E., Quinkler, T., and De Renzis, S. (2018). Guided morphogenesis through optogenetic activation of Rho signalling during early Drosophila embryogenesis. Nat Commun 9, 2366.

96. Gupta, T., and Schupbach, T. (2003). Cct1, a phosphatidylcholine biosynthesis enzyme, is required for Drosophila oogenesis and ovarian morphogenesis. Development 130, 6075-6087.

97. Besson, C., Bernard, F., Corson, F., Rouault, H., Reynaud, E., Keder, A., Mazouni, K., and Schweisguth, F. (2015). Planar Cell Polarity Breaks the Symmetry of PAR Protein Distribution prior to Mitosis in Drosophila Sensory Organ Precursor Cells. Curr Biol 25, 11041110.

98. Xu, T., and Rubin, G.M. (1993). Analysis of genetic mosaics in developing and adult Drosophila tissues. Development 117, 1223-1237.

99. Betschinger, J., Mechtler, K., and Knoblich, J.A. (2006). Asymmetric segregation of the tumor suppressor brat regulates self-renewal in Drosophila neural stem cells. Cell 124, 1241-1253.

100. Schindelin, J., Arganda-Carreras, I., Frise, E., Kaynig, V., Longair, M., Pietzsch, T., Preibisch, S., Rueden, C., Saalfeld, S., Schmid, B., et al. (2012). Fiji: an open-source platform for biological-image analysis. Nat Methods 9, 676-682.

101. Jia, D., Xu, Q., Xie, Q., Mio, W., and Deng, W.M. (2016). Automatic stage identification of Drosophila egg chamber based on DAPI images. Sci Rep 6, 18850. 